\title{
Two lectures on color superconductivity*
}

\author{
Igor A. Shovkovy ${ }^{\dagger}$ \\ Frankfurt Institute for Advanced Studies \\ and \\ Institut für Theoretische Physik, \\ Johann Wolfgang Goethe-Universität, \\ D-60054 Frankurt am Main, Germany
}

February 4, 2008

\begin{abstract}
The first lecture provides an introduction to the physics of color superconductivity in cold dense quark matter. The main color superconducting phases are briefly described and their properties are listed. The second lecture covers recent developments in studies of color superconducting phases in neutral and $\beta$-equilibrated matter. The properties of gapless color superconducting phases are discussed.
\end{abstract}

${ }^{*}$ Lectures delivered at the IARD 2004 conference, Saas Fee, Switzerland, June 12 - 19, 2004, and at the Helmholtz International Summer School and Workshop on Hot points in Astrophysics and Cosmology, JINR, Dubna, Russia, August 2 - 13, 2004.

${ }^{\dagger}$ On leave from Bogolyubov Institute for Theoretical Physics, 03143, Kiev, Ukraine. 


\section{Contents}

1 Introduction into color superconductivity 3

1.1 Dense barvonic matter . . . . . . . . . . . . . . . . . . 3

1.1.1 Weaklv interacting auark matten ... . . . . . . . 4

1.2 Cooper instability and color superconductivity . . . . . . . 5

1.3 Two-flavor color superconductivity $\left(N_{f}=2\right) \ldots \ldots \ldots 7$

1.3.1 Color and flavor structure of the condensate . . . . . 10

1.3.2 Quark propagator in the Nambu-Gorkov basis . . . . . 11

1.3 .3 One-gluon exchange interaction . . . . . . . . . . . 12

1.3.4 Gap equation in QCD . . . . . . . . . . . . . . 15

1.3.5 Gap equation in the NJL model . . . . . . . . . . . 17

1.3.6 Properties of quark matter in the 2SC phase . . . . . . 19

1.4 Color-flavor locked phase $\left(N_{f}=3\right) \ldots \ldots \ldots \ldots . \ldots 21$

1.4.1 Properties of quark matter in the CFL phase . . . . . . 23

1.4 .2 Low-energy effective action . . . . . . . . . . . . . . 24

1.5 Spin-1 color superconductivity $\left(N_{f}=1\right) \ldots \ldots \ldots$

1.6 Summarv of the first lecture . . . . . . . . . . . . . 27

2 Color superconductivity in neutral matter 29

2.1 Dense matter inside stars . . . . . . . . . . . . . . . . . . . 29

2.2 Gapless two-flavor color superconductivity . . . . . . . . . . . 30

2.2 .1 Neutrality vs. color superconductivity . . . . . . . . 30

2.2 .2 Model . . . . . . . . . . . . . . . . . . . . . . 31

2.2 .3 Effective potential . . . . . . . . . . . . . . 33

2.2 .4 Three regimes in neutral matter . . . . . . . . . . . 35

2.2 .5 Gapless 2SC phase . . . . . . . . . . . . . . . . . 37

2.2 .6 Finite temperature properties . . . . . . . . . . . 39

2.3 Gapless color-flavor locked phase ... . . . . . . . . . . . 40

2.4 Mixed phases with color superconducting components . . . . . 44

2.4 .1 Gibbs construction ................. . . . 44

2.4.2 Surface tension and Coulomb forces . . . . . . . . . . 46

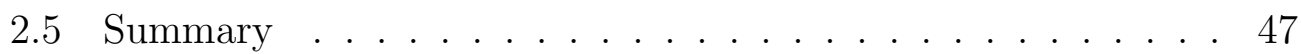




\section{Introduction into color superconductivity}

\subsection{Dense baryonic matter}

Almost all matter around us is made of several dozen chemical elements and their isotopes. Each element (atom) has a compact nucleus, made of protons and neutrons, and a cloud of electrons surrounding the nucleus. The proton and the neutron are baryons, i.e., "heavy" particles. They are about two thousand times heavier than the electron. (The actual values of the masses are $m_{\mathrm{p}} \approx 938.3 \mathrm{MeV} / c^{2}, m_{\mathrm{n}} \approx 939.6 \mathrm{MeV} / c^{2}$, and $m_{\mathrm{e}} \approx 0.511 \mathrm{MeV} / c^{2}$, see Ref. [1.) Thus, the mass of an atom comes mostly from its heavy nucleus. A typical size of a nucleus is about five orders of magnitude smaller than a typical atomic size. It is clear, therefore, that the density of matter inside a nucleus is much larger than the density of matter made of chemical elements. In fact, typical densities inside nuclei are of order $10^{14} \mathrm{~g} / \mathrm{cm}^{3}$. To get an impression how large this is, it suffices to say that a tablespoon of a material with this density would weigh about a billion tonnes. Creating even a tiny macroscopic sample of such matter in laboratory is impossible. On the other hand, baryonic matter at even higher densities exists in the Universe in the interior of compact stars. Therefore, it is of fundamental importance to study such very dense matter.

Protons and neutrons, as well as all mesons and baryons that may appear in dense matter, are strongly interacting particles. Their properties and the properties of dense baryonic matter are, in principle, described by the microscopic theory of strong interactions, Quantum Chromodynamics (QCD). The only problem is that QCD is a rather complicated non-Abelian gauge theory. The Lagrangian density of the theory is written in terms of quark fields which carry color charges. Because of the property of confinement, however, quarks cannot exist in vacuum as free particles. Instead, they combine with other quarks and antiquarks to form color neutral hadrons (i.e., mesons and baryons) whose description is not easy in the framework of QCD.

It is known that baryons are not point-like particles. They have a typical size of about $1 \mathrm{fm}=10^{-13} \mathrm{~cm}$. In sufficiently dense matter, therefore, baryons can be forced to stay so close to one another that they would overlap. At such densities, constituent quarks are shared by neighboring baryons and, with increasing the density further, quarks should eventually become mobile over large distances. This means that quarks become deconfined. When this happens, it does not make sense to talk about hadronic matter any more. It 
is transformed into quark matter. The properties of such matter is the topic of these lectures.

It was suggested long time ago that quark matter may exist inside central regions of compact stars [2]. By making use of the property of asymptotic freedom in QCD [3], it was argued that quarks interact weakly, and that realistic calculations taking full account of strong interactions are possible for sufficiently dense matter 4]. The argument of Ref. 4] consisted of the two main points: (i) the long-range QCD interactions are screened in dense medium causing no infrared problems, and (ii) at short distances, the interaction is weak enough to allow the use of the perturbation theory. As we shall see below, the real situation in dense quark matter is slightly more subtle.

\subsubsection{Weakly interacting quark matter}

By assuming that very dense matter is indeed made of weakly interacting quarks, one could try to understand the thermodynamic properties of the corresponding ground state by first completely neglecting the interaction between quarks. In order to construct the ground state, it is important to keep in mind that quarks are fermions, i.e., particles with a half-integer spin, $s=1 / 2$. Therefore, quarks should obey the Pauli exclusion principle which says that no two identical quarks can simultaneously occupy the same quantum state.

In the ground state of zero temperature non-interacting quark matter, quarks occupy all available quantum states with the lowest possible energies. This is described formally by the following quark distribution function:

$$
f_{F}(\mathbf{k})=\theta\left(\mu-E_{\mathbf{k}}\right), \quad \text { at } \quad T=0,
$$

where $\mu$ is the quark chemical potential, and $E_{\mathbf{k}} \equiv \sqrt{k^{2}+m^{2}}$ is the energy of a free quark (with mass $m$ ) in the quantum state with the momentum $\mathbf{k}$ (by definition, $k \equiv|\mathbf{k}|$ ). As one can see, $f_{F}(\mathbf{k})=1$ for the states with $k<k_{F} \equiv \sqrt{\mu^{2}-m^{2}}$, indicating that all states with the momenta less than the Fermi momentum $k_{F}$ are occupied. The states with the momenta greater than the Fermi momentum $k_{F}$ are empty, i.e., $f_{F}(\mathbf{k})=0$ for $k>k_{F}$.

In the chiral limit, i.e., when the quark mass is vanishing, the pressure of zero temperature free quark matter is given by [5]

$$
P^{(0)}=2 N_{f} N_{c} \int \frac{d^{3} \mathbf{k}}{(2 \pi)^{3}}\left(\mu-E_{\mathbf{k}}\right) \theta\left(\mu-E_{\mathbf{k}}\right)-B=\frac{N_{f} N_{c}}{12 \pi^{2}} \mu^{4}-B,
$$


where the overall factor $2 N_{f} N_{c}$ counts the total number of degenerate quark states, namely 2 spin states (i.e., $s= \pm 1 / 2$ ), $N_{f}$ flavor states (e.g., up, down and strange), and $N_{c}$ color states (e.g., red, green and blue). The extra term $B$ in the expression for the pressure (2), called the bag constant, was added by hand. This term effectively assigns a nonzero contribution to the vacuum pressure and, in this way, provides the simplest modelling of the quark confinement in QCD [6]. [Note that the vacuum pressure is higher than the pressure of quark matter when $\mu<\left(4 \pi^{2} B / N_{f}\right)^{1 / 4}$.] Here, we ignore the requirements of the charge neutrality and the $\beta$ equilibrium in quark matter. These will be addressed in detail in the second lecture. The energy density of quark matter reads

$$
\epsilon^{(0)} \equiv \mu \frac{\partial P^{(0)}}{\partial \mu}-P^{(0)}=\frac{N_{f} N_{c}}{4 \pi^{2}} \mu^{4}+B .
$$

Thus, one arrives at the following equation of state:

$$
P^{(0)}=\frac{1}{3}\left(\epsilon^{(0)}-4 B\right) \text {. }
$$

This equation of state could be easily generalized to the case of nonzero quark masses and/or to the case of non-equal chemical potentials for different quarks. Also, it could be further improved by adding the lowest order corrections due to the interaction. For example, to leading order in coupling, the interaction results in the following correction to the pressure [7]:

$$
\delta P^{(0)}=-\frac{\alpha_{s} N_{f}\left(N_{c}^{2}-1\right)}{16 \pi^{3}} \mu^{4}+O\left(\alpha_{s}^{2}\right),
$$

where $\alpha_{s} \equiv g^{2} / 4 \pi$ is the value of the running coupling constant of strong interactions defined at the scale of the quark chemical potential. The next to leading order corrections can also be calculated [7.

It is generally believed that the equation of state in Eq. (2), or its generalization, provides a good approximation for the description of weakly interacting quark matter. Below we argue, however, that some underlying assumptions regarding the ground state of such quark matter are not correct.

\subsection{Cooper instability and color superconductivity}

It appears that the perturbative ground state of quark matter, characterized by the distribution function in Eq. (11), is unstable when there is an attractive 
(even arbitrarily weak in magnitude!) interaction between quarks. This is because of the famous Cooper instability [8] that develops as a result of the formation of Cooper pairs $\left\langle q_{\mathbf{k}} q_{-\mathbf{k}}\right\rangle$ made of quarks from around the highly degenerate Fermi surface, i.e., quarks with the absolute value of momenta $k \simeq k_{F}$. Cooper pairs are bosons, and they occupy the same lowest energy quantum state at zero temperature, producing a Bose condensate. In the presence of such a condensate of Cooper pairs, the ground state of quark matter becomes a (color) superconductor. This is very similar to the ground state of the electron system in the Bardeen-Cooper-Schrieffer theory of lowtemperature superconductivity [9]. The only real difference comes from the fact that quarks, unlike electrons, come in various flavors (e.g., up, down and strange) and carry non-Abelian color charges. To emphasize this difference, superconductivity in quark matter is called color superconductivity. For recent reviews on color superconductivity, see Ref. [10].

It was known for a long time that dense quark matter should be a color superconductor [11, 12. In many studies, however, this fact was commonly ignored. Only recently, the potential importance of this phenomenon was appreciated. To large extent, this was triggered by the observation in Ref. [13] that the value of the color superconducting gap could be as large as $100 \mathrm{MeV}$ at baryon densities existing in the central regions of compact stars, i.e., at densities which are a few times larger than the normal nuclear density, $n_{0} \simeq 0.15 \mathrm{fm}^{-3}$. This very natural estimate for the value of the gap in QCD, in which a typical energy scale itself is $200 \mathrm{MeV}$, opened a wide range of new theoretical possibilities, and the subject bursted with numerous studies. The main reason is that the presence of such a large energy gap in the quark spectrum may allow to extract clear signatures of color superconducting states of matter in observational data from compact stars.

As in low-temperature superconductors, one of the main consequences of color superconductivity in dense quark matter is the appearance of a nonzero energy gap in the one-particle spectrum,

$$
\mathcal{E}_{\mathbf{k}}=\sqrt{\left(E_{\mathbf{k}}-\mu\right)^{2}+\Delta^{2}},
$$

where $\Delta$ is the gap. The presence of the gap in the energy spectrum should affect transport properties (e.g., conductivities and viscosities) of quark matter. Thus, if quark matter exists in the interior of compact stars, this will be reflected, for example, in the cooling rates and in the rotational slowing down of such stars. Also, a nonzero gap modifies thermodynamic properties, 
e.g., the specific heat and the equation of state. In application to stars, this could modify theoretical predictions for the mass-radius relations, or even suggest the existence of a new family of compact stars.

Color superconductivity may affect directly as well as indirectly many other observed properties of stars. In some cases, for example, superconductivity may be accompanied by the baryon superfluidity and/or the electromagnetic Meissner effect. If matter is superfluid, rotational vortices would be formed in the stellar core, and they would carry a portion of the angular momentum of the star. Because of the Meissner effect, the star interior could become threaded with magnetic flux tubes. In either case, the star evolution may be affected.

In general, it is of great phenomenological interest to perform a systematic study of all possible effects of color superconductivity in compact stars. Before this can be done, however, one needs to know the structure of the QCD phase diagram and properties of various color superconducting phases in detail. Despite the recent progress in the field, such knowledge still remains patchy. While many different phases of quark matter have been proposed, there is no certainty that all possibilities have already been exhausted. This is especially so when additional requirements of charge neutrality and $\beta$ equilibrium are imposed.

In the rest of this lecture, the main color superconducting phases, that involve different number of pairing quark flavors, will be reviewed briefly. The role of charge neutrality and $\beta$ equilibrium in quark matter will be discussed in the second lecture.

\subsection{Two-flavor color superconductivity $\left(N_{f}=2\right)$}

It is instructive to start this lecture from the description of the simplest color superconducting phase, namely the two-flavor color superconductor (2SC). This is a color superconducting phase with the spin-0 Cooper pairing in quark matter made of up and down quarks.

As we discussed in the previous subsection, color superconductivity comes as a result of the Cooper instability driven by an attractive interaction between quarks. To study this instability in detail, one needs to specify the origin and the strength of the interaction. At very large densities, when quark matter is weakly interacting, the dominant interaction between quarks is provided by the one-gluon exchange. Then, the Cooper pairing dynamics, including the screening effects due to dense medium as well as the effects due 
to higher order corrections, can be studied from first principles within the framework of QCD. Some key details of the analysis in this regime are given below.

We consider quark fields $\psi_{i}^{a}$ in the fundamental representation of the $\mathrm{SU}(3)_{c}$ color gauge group. These fields carry flavor $(i=1,2)$ and color $(a=1,2,3)$ indices. The QCD Lagrangian density reads

$$
\mathcal{L}_{\mathrm{QCD}}=\bar{\psi}_{i}^{a}\left(i \gamma^{\mu} \partial_{\mu}+\gamma^{0} \mu-m_{i}^{(0)}\right) \psi_{i}^{a}+g A_{\mu}^{A} \bar{\psi}_{i}^{a} \gamma^{\mu} T_{a b}^{A} \psi_{i}^{b}-\frac{1}{4} G_{\mu \nu}^{A} G^{A, \mu \nu},
$$

where $A_{\mu}^{A}$ is the vector gauge field in the adjoint representation of $\mathrm{SU}(3)_{c}$, $G_{\mu \nu}^{A}=\partial_{\mu} A_{\nu}^{A}-\partial_{\nu} A_{\mu}^{A}+g f^{A B C} A_{\mu}^{B} A_{\nu}^{C}$ is the field strength, and the generators of color transformations are defined as $T_{a b}^{A} \equiv \frac{1}{2}\left(\lambda^{A}\right)_{a b}$ where $\lambda^{A}$ are the GellMann matrices. The current quark masses and the quark chemical potential are denoted by $m_{i}^{(0)}$ and $\mu$, respectively. At high densities, one can neglect small current masses of quarks. Then, the QCD Lagrangian density (7) becomes invariant under $\mathrm{SU}(2)_{L} \times \mathrm{SU}(2)_{R}$ global chiral transformations.

In QCD, the constituent masses of quarks $m_{i}$ are generated dynamically, and they can be very different from the current masses $m_{i}^{(0)}$, appearing in the Lagrangian density. At zero density, for example, typical values of the constituent quark masses are of order $\frac{1}{3} m_{\mathrm{n}} \approx 313 \mathrm{MeV}$ even in the chiral limit. At high densities, on the other hand, the masses of the up and down quarks become small. This is because they are proportional to the value of the chiral condensate $\left\langle\bar{\psi}_{L} \psi_{R}\right\rangle$ which melts in dense matter. Thus, it is often also justified to neglect the constituent quark masses in studies of color superconducting phases.

The interaction vertex in the Lagrangian density (17) has a non-trivial color structure given by the color generators $T_{a b}^{A}$. As a result, the quarkquark scattering amplitude in the one-gluon exchange approximation is proportional to the following color tensor:

$$
\sum_{A=1}^{N_{c}^{2}-1} T_{a a^{\prime}}^{A} T_{b^{\prime} b}^{A}=-\frac{N_{c}+1}{4 N_{c}}\left(\delta_{a a^{\prime}} \delta_{b^{\prime} b}-\delta_{a b^{\prime}} \delta_{a^{\prime} b}\right)+\frac{N_{c}-1}{4 N_{c}}\left(\delta_{a a^{\prime}} \delta_{b^{\prime} b}+\delta_{a b^{\prime}} \delta_{a^{\prime} b}\right) .
$$

The first antisymmetric term corresponds to the attractive antitriplet channel, while the second symmetric term corresponds to the repulsive sextet channel, see Fig. 1. It is the first antisymmetric antitriplet channel that plays the crucial role in Cooper pairing. 


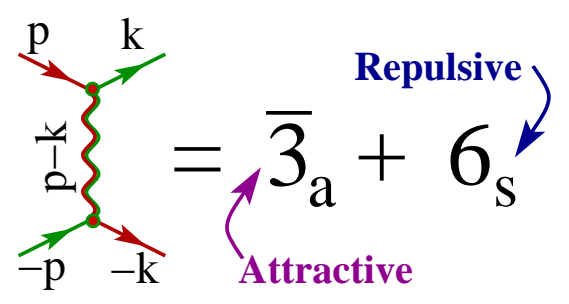

Figure 1: The diagrammatic representation of the one-gluon exchange interaction between two quarks in QCD. The color structure of the corresponding amplitude contains an antisymmetric antitriplet and a symmetric sextet channel.

Here, it is appropriate to note that quark matter is unlikely to be truly weakly interacting at moderate densities existing in the central regions of compact stars, i.e., $n \lesssim 10 n_{0}$. In this case, the use of the microscopic theory of strong interactions is very limited, and one has to rely on various effective models of QCD. A very simple type of such a model is the Nambu-JonaLasinio (NJL) model with a local four-fermion interaction [14. Originally, this model was introduced for the description of nucleons with dynamically generated masses. Nowadays, it is also commonly used for the description of quarks. For example, the NJL type models were used in Refs. [13, 15, 16, 17] for the description of color superconducting phases. One of the simplest NJL models, that respects the $\mathrm{SU}(2)_{L} \times \mathrm{SU}(2)_{R}$ global chiral symmetry (in the limit $m_{i}^{(0)} \rightarrow 0$ ), is defined by the following Lagrangian density [18]:

$$
\begin{aligned}
\mathcal{L}_{\mathrm{NJL}} & =\bar{\psi}_{i}^{a}\left(i \gamma^{\mu} \partial_{\mu}+\gamma^{0} \mu-m_{i}^{(0)}\right) \psi_{i}^{a}+G_{S}\left[(\bar{\psi} \psi)^{2}+\left(i \bar{\psi} \gamma_{5} \vec{\tau} \psi\right)^{2}\right] \\
& +G_{D}\left(i \bar{\psi}^{C} \varepsilon \epsilon^{a} \gamma_{5} \psi\right)\left(i \bar{\psi} \varepsilon \epsilon^{a} \gamma_{5} \psi^{C}\right),
\end{aligned}
$$

where $\psi^{C}=C \bar{\psi}^{T}$ is the charge-conjugate spinor and $C=i \gamma^{2} \gamma^{0}$ is the charge conjugation matrix. The matrix $C$ is defined so that $C \gamma_{\mu} C^{-1}=-\gamma_{\mu}^{T}$. Regarding the other notation, $\vec{\tau}=\left(\tau^{1}, \tau^{2}, \tau^{3}\right)$ are the Pauli matrices in the flavor space, while $(\varepsilon)^{i k} \equiv \varepsilon^{i k}$ and $\left(\epsilon^{a}\right)^{b c} \equiv \epsilon^{a b c}$ are the antisymmetric tensors in the flavor and in the color spaces, respectively. The dimensionful coupling constant $G_{S}=5.01 \mathrm{GeV}^{-2}$ and the momentum integration cutoff parameter $\Lambda=0.65 \mathrm{GeV}$ (which appears only in loop calculations) are adjusted so that the values of the pion decay constant and the value of the chiral condensate take their standard values in vacuum QCD: $F_{\pi}=93 \mathrm{MeV}$ and $\langle\bar{u} u\rangle=\langle\bar{d} d\rangle=(-250 \mathrm{MeV})^{3}$ [18]. The strength of the coupling constant 
$G_{D}$ is taken to be proportional to the value of $G_{S}$ as follows: $G_{D}=\eta G_{S}$ where $\eta$ is a dimensionless parameter of order 1 . Note that the value of $\eta$ is positive which corresponds to having the antisymmetric diquark channel attractive. This is motivated by the microscopic QCD interaction, as well as by the instanton motivated models [13].

\subsubsection{Color and flavor structure of the condensate}

Now let us discuss the color and flavor structure of the condensate of Cooper pairs in the 2SC ground state. This can be determined from the nature of the diquark interaction and the Pauli exclusion principle.

The color structure of the one-gluon exchange interaction in Eq. (8) shows that the color antisymmetric antitriplet channel is attractive. The antisymmetric color channel is also attractive in the instanton motivated models of Ref. [13. This is reflected in the color structure of the condensate of spin-0 Cooper pairs,

$$
\left\langle\left(\bar{\psi}^{C}\right)_{i}^{a} \gamma^{5} \psi_{j}^{b}\right\rangle \sim \varepsilon_{i j} \epsilon^{a b c},
$$

which is antisymmetric in the color indices of the constituent quarks. As one can check (not shown explicitly), this spin-0 condensate is also antisymmetric in Dirac indices. Then, in accordance with the Pauli exclusion principle, it should be antisymmetric in flavor indices as well. The presence of the $\gamma^{5}$ matrix on the left hand side of Eq. (10) makes the condensate a scalar. A similar condensate without $\gamma^{5}$ is a pseudoscalar which is disfavored in QCD because of the instanton effects.

The color antitriplet condensate (10) may have an arbitrary orientation in the color space. By making use of the global color transformations, this orientation can be changed as convenient. It is conventional to choose the condensate to point in the third (blue) color direction, $\left\langle\left(\bar{\psi}^{C}\right)_{i}^{a} \gamma^{5} \psi_{j}^{b}\right\rangle \sim \varepsilon_{i j} \epsilon^{a b 3}$. In this case, the Cooper pairs in the 2SC phase are made of the red and green quarks only, and the blue quarks do not participate in the pairing. These unpaired blue quarks give rise to gapless quasiparticles in the low-energy effective theory.

The flavor antisymmetric structure in Eq. (10) corresponds to a singlet representation of the global $\mathrm{SU}(2)_{L} \times \mathrm{SU}(2)_{R}$ chiral group. This means that the chiral symmetry is not broken in the 2SC ground state. In fact, there are no other global continuous symmetries which are broken in the 2SC phase. There exist, however, several approximate symmetries which are broken. One 
of them is the approximate $\mathrm{U}(1)_{A}$ symmetry which is a good symmetry at high density when the instantons are screened [19]. Its breaking in the 2SC phase results in a pseudo-Nambu-Goldstone boson [20]. Additional four exotic pseudo-Nambu-Goldstone states may appear as a result of a less obvious approximate axial color symmetry discussed in Ref. 21].

In the $2 \mathrm{SC}$ ground state, the vector-like $\mathrm{SU}(3)_{c}$ color gauge group of $\mathrm{QCD}$ is broken down to the $\mathrm{SU}(2)_{c}$ subgroup. Therefore, five out of total eight gluons of $\mathrm{SU}(3)_{c}$ become massive due to the Anderson-Higgs mechanism. The other three gluons, which correspond to the unbroken $\mathrm{SU}(2)_{c}$, do not interact with the gapless blue quasiparticles. They give rise to a pure $\mathrm{SU}(2)_{c}$ gluodynamics. The red and green quasiparticles decouple from this lowenergy $\mathrm{SU}(2)_{c}$ gluodynamics because they are gapped [22].

\subsubsection{Quark propagator in the Nambu-Gorkov basis}

To simplify the study of diquark condensates in dense quark matter, instead of using the Dirac spinors for quark fields, it is more convenient to introduce the following eight-component Nambu-Gorkov spinors:

$$
\Psi \equiv\left(\begin{array}{l}
\psi \\
\psi^{C}
\end{array}\right),
$$

where $\psi^{C}$ is the charge-conjugate spinor, defined earlier. The structure of the inverse quark propagator in this basis reads

$$
S_{0}^{-1}=\left(\begin{array}{cc}
{\left[G_{0}^{+}\right]^{-1}} & 0 \\
0 & {\left[G_{0}^{-}\right]^{-1}}
\end{array}\right),
$$

where

$$
\left[G_{0}^{ \pm}\right]^{-1}=\gamma^{\mu} K_{\mu} \pm \mu \gamma_{0}
$$

are the inverse Dirac propagators for massless quarks $\left(G_{0}^{+}\right)$and chargeconjugate quarks $\left(G_{0}^{-}\right)$.

In the ground state with Cooper pairing, see Eq. (10), the quark propagator in the Nambu-Gorkov basis also has nonzero off-diagonal components,

$$
S^{-1}=\left(\begin{array}{cc}
{\left[G_{0}^{+}\right]^{-1}} & \Delta^{-} \\
\Delta^{+} & {\left[G_{0}^{-}\right]^{-1}}
\end{array}\right),
$$


where $\Delta^{-}=-i \epsilon^{3} \varepsilon \gamma^{5} \Delta$ and $\Delta^{+} \equiv \gamma^{0}\left(\Delta^{-}\right)^{\dagger} \gamma^{0}=-i \epsilon^{3} \varepsilon \gamma^{5} \Delta^{*}$ are the matrices in the Dirac space, and $\Delta$ is the diquark gap parameter. This is the simplest ansatz for the quark propagator in the color superconducting ground state. It can be made more general, for example, by adding a regular (diagonal) part of the quark self-energy. Such a self-energy plays an essential role in the dynamics of chiral symmetry breaking. In dense quark matter, on the other hand, it is not so important. Thus, for simplicity, it is neglected here (the effects of the diagonal part of the self-energy were studied in Ref. [23]).

From the inverse propagator in Eq. (14), one can derive the following expression for the quark propagator:

$$
S=\left(\begin{array}{cc}
G^{+} & \Xi^{-} \\
\Xi^{+} & G^{-}
\end{array}\right),
$$

where

$$
\begin{aligned}
G^{ \pm} & =\left[\left(G_{0}^{ \pm}\right)^{-1}-\Delta^{\mp} G_{0}^{\mp} \Delta^{ \pm}\right]^{-1} \\
\Xi^{ \pm} & =-G_{0}^{\mp} \Delta^{ \pm} G^{ \pm}
\end{aligned}
$$

This structure of the quark propagator carries basic information about the properties of the 2SC ground state. For instance, the location of its poles in the energy plane gives the quasiparticle dispersion relations.

The only quantity that remains unknown in the ansatz (14) is the value of the gap parameter $\Delta$. This should be determined from a self-consistent gap equation that takes into account the pairing dynamics between quarks. This is discussed in more detail in the next three subsections.

\subsubsection{One-gluon exchange interaction}

In dense QCD, in which quarks are weakly interacting, the one-gluon exchange is the dominant interaction between quarks. It appears, however, that the one-gluon interaction is partially screened by surrounding dense medium. Thus, before studying the pairing dynamics between quarks, we first address the modification of gluon properties due to dense medium.

The inverse propagator of the medium modified gluon can be written in the following form:

$$
\left(\mathcal{D}^{-1}\right)_{\mu \nu}^{A B}=\left(D_{0}^{-1}\right)_{\mu \nu}^{A B}+\Pi_{\mu \nu}^{A B}
$$


where $\Pi_{\mu \nu}^{A B}$ is the gluon self-energy, or the polarization tensor. The gluons with soft momenta, $p \lesssim g_{s} \mu$, play the key role in Cooper pairing of quarks. The main contribution to the corresponding soft gluon polarization tensor comes from the quark loop with hard internal momenta of order $\mu$. This contribution is large compared to all other contributions in QCD (e.g., those from the ghost and the gluon loops) because it is proportional to the density of quark states at the Fermi surface (as well as to the coupling constant $\alpha_{s}$ ), i.e., $\Pi_{\mu \nu}^{A B} \sim \alpha_{s} \mu^{2}$. The corresponding approximation, in which the subleading terms suppressed by powers of the quark chemical potential $\mu$ are neglected, is called the hard dense loop (HDL) approximation.

The calculation of the polarization tensor in the HDL approximation was performed in Refs. 24, 25] (in the case of dense QCD in $2+1$ dimensions, similar calculations were performed in Ref. [26]). Here, we present the final result and briefly comment on its main features.

The HDL polarization tensor can be written as follows: $\Pi_{\mu \nu}^{A B} \equiv \delta^{A B} \Pi_{\mu \nu}$, where the explicit form of the components of $\Pi_{\mu \nu}$ are given by

$$
\begin{aligned}
\Pi^{00}\left(p_{0}, \mathbf{p}\right) & =\Pi_{l}\left(p_{0}, \mathbf{p}\right), \\
\Pi^{0 i}\left(p_{0}, \mathbf{p}\right) & =p_{0} \frac{p^{i}}{p^{2}} \Pi_{l}\left(p_{0}, \mathbf{p}\right), \\
\Pi^{i j}\left(p_{0}, \mathbf{p}\right) & =\left(\delta^{i j}-\frac{p^{i} p^{j}}{p^{2}}\right) \Pi_{t}\left(p_{0}, \mathbf{p}\right)+\frac{p^{i} p^{j}}{p^{2}} \frac{p_{0}^{2}}{p^{2}} \Pi_{l}\left(p_{0}, \mathbf{p}\right),
\end{aligned}
$$

with

$$
\begin{aligned}
& \Pi_{l}\left(p_{0}, \mathbf{p}\right)=m_{D}^{2}\left(\frac{p_{0}}{2 p} \ln \left|\frac{p_{0}+p}{p_{0}-p}\right|-1-i \pi \frac{p_{0}}{2 p} \theta\left(-p^{2}\right)\right) \\
& \Pi_{t}\left(p_{0}, \mathbf{p}\right)=\frac{1}{2} m_{D}^{2}-\frac{P^{2}}{2 p^{2}} \Pi_{l}\left(p_{0}, \mathbf{p}\right) .
\end{aligned}
$$

Here, we use the notation $p^{2} \equiv \mathbf{p}^{2}, P^{2}=p_{0}^{2}-p^{2}$, and $m_{D}^{2} \equiv 2 N_{f} \alpha_{s} \mu^{2} / \pi$. It is remarkable that this result coincides with the polarization tensor derived in the framework of the classical transport theory of dense Yang-Mills plasma 27. One can check that the HDL polarization tensor is transverse, i.e.,

$$
P^{\mu} \Pi_{\mu \nu}\left(p_{0}, \mathbf{p}\right)=0 .
$$

It is convenient to write the tensor $\Pi_{\mu \nu}$ in terms of two transverse projection operators,

$$
\Pi_{\mu \nu}=-O_{\mu \nu}^{(1)} \Pi_{t}+O_{\mu \nu}^{(2)}\left(2 \Pi_{t}-m_{D}^{2}\right) .
$$


where

$$
\begin{aligned}
O_{\mu \nu}^{(1)} & =g_{\mu \nu}-u_{\mu} u_{\nu}+\frac{\mathbf{p}_{\mu} \mathbf{p}_{\nu}}{p^{2}} \\
O_{\mu \nu}^{(2)} & =u_{\mu} u_{\nu}-\frac{\mathbf{p}_{\mu} \mathbf{p}_{\nu}}{p^{2}}-\frac{P_{\mu} P_{\nu}}{P^{2}},
\end{aligned}
$$

and $u_{\mu}=(1,0,0,0)$ is the time-like four-vector that defines the rest frame of dense quark medium. In order to have a complete set of projectors such that $\sum_{I} O_{\mu \nu}^{(I)}=g_{\mu \nu}$, one has to include also the following longitudinal projector:

$$
O_{\mu \nu}^{(3)}=\frac{P_{\mu} P_{\nu}}{P^{2}}
$$

By making use of this set of three projectors, inverse gluon propagator in Eq. (18) can be represented as follows:

$$
\left(\mathcal{D}^{-1}\right)_{\mu \nu}^{A B}=i \delta^{A B}\left[\left(P^{2}-\Pi_{t}\right) O_{\mu \nu}^{(1)}+\left(P^{2}+2 \Pi_{t}-m_{D}^{2}\right) O_{\mu \nu}^{(2)}+\frac{P^{2}}{\lambda} O_{\mu \nu}^{(3)}\right],
$$

where $\lambda$ is the gauge fixing parameter. This expression can be easily inverted, leading to the following result for the gluon propagator:

$$
\mathcal{D}_{\mu \nu}^{A B}=-i \delta^{A B}\left(\frac{1}{P^{2}-\Pi_{t}} O_{\mu \nu}^{(1)}+\frac{1}{P^{2}+2 \Pi_{t}-m_{D}^{2}} O_{\mu \nu}^{(2)}+\frac{\lambda}{P^{2}} O_{\mu \nu}^{(3)}\right) .
$$

The gluon modes which are transverse and longitudinal with respect to the three-momentum $\mathbf{p}$ are called the magnetic and electric modes, respectively. These two types of physical gluon modes are described by the following fields:

$$
\begin{aligned}
a_{\mu}^{(\mathrm{mag})} & =O_{\mu \nu}^{(1)} A^{\nu}, \\
a_{\mu}^{(\mathrm{el})} & =O_{\mu \nu}^{(2)} A^{\nu} .
\end{aligned}
$$

From Eq. (30) one can see that the propagators of these modes are naturally separated from each other, as well as from the unphysical longitudinal mode, described by the field $a_{\mu}^{(\|)}=O_{\mu \nu}^{(3)} A^{\nu}$.

In the study of the Cooper pairing dynamics, the most relevant part of the one-gluon exchange interaction is the interaction with the space-like momenta such that $p_{0} \ll p$. This should be clear from the kinematics of the quark scattering around the Fermi surface: the energy exchange between 
pairing quarks is typically much smaller than the change of their momenta. It is justified, therefore, to use the approximate form of $\Pi_{t}$, obtained in the region $p_{0} \ll p$,

$$
\Pi_{t} \simeq m_{D}^{2}\left(i \pi \frac{p_{0}}{4 p} \theta\left(P^{2}\right)+\frac{p_{0}^{2}}{p^{2}}+\ldots\right)
$$

By substituting this asymptote into the gluon propagator (30), one can check that the magnetic modes produce a long-range (dynamically screened) interaction, while the electric mode works only at short distances, $r \lesssim 1 / m_{D}$.

In the next subsection, the HDL gluon propagator is used in the study of the Cooper pairing dynamics. As we shall see there, the long-range interaction mediated by the magnetic modes will play a particularly important role in the dynamics.

\subsubsection{Gap equation in QCD}

Color superconductivity is an essentially non-perturbative phenomenon which cannot be addressed with perturbative techniques even if the theory is weakly interacting. The standard method for studying superconductivity is the method of the Schwinger-Dyson equations. In general, the complete set of Schwinger-Dyson equations contains an infinite number of coupled equations for Green's functions. There exist no systematic ways of solving such equations exactly. However, there exist various standard approximations that can be justified if the dynamics is controlled by a small parameter (e.g., a weak coupling constant, or a small $1 / N$ parameter where $N$ is the number of flavors or colors in the model).

In the case of QCD at asymptotic densities, the main features of its dynamics are well described by the so-called improved rainbow approximation. In this approximation, one uses the bare quark-gluon vertices in the Schwinger-Dyson equation for the quark propagator. One also uses the gluon propagator with the screening effects taken into account in the HDL approximation. (Note that, in the simple rainbow approximation, the screening is usually neglected.) The graphical representation of the Schwinger-Dyson equation is shown in Fig. 2. The analytical form of this equation reads

$$
S^{-1}(K)=S_{0}^{-1}(K)+4 \pi \alpha_{s} \int \frac{d^{4} p}{(2 \pi)^{4}} \Gamma_{\mu}^{A} S(P) \Gamma_{\nu}^{B} \mathcal{D}_{A B}^{\mu \nu}(K-P)
$$




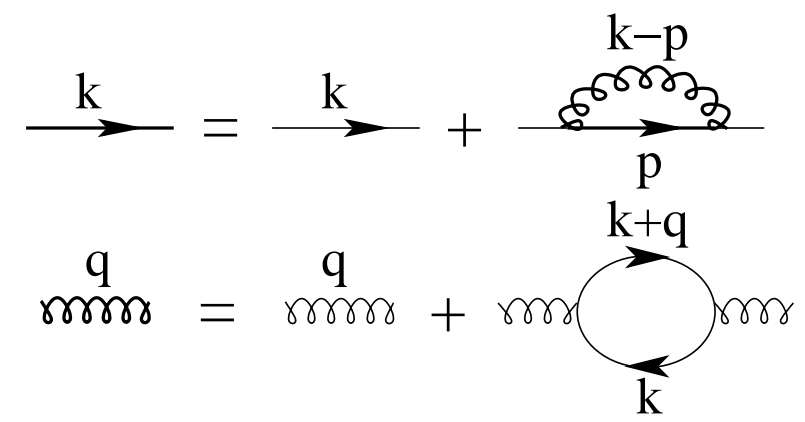

Figure 2: The graphical representation of the Schwinger-Dyson equation in the improved rainbow approximation. The quark propagator in the NambuGorkov basis and the gluon propagator are denoted by the solid lines and the wavy lines, respectively.

where the quark-gluon vertices in the Nambu-Gorkov basis are given by

$$
\Gamma_{\mu}^{A}=\gamma_{\mu}\left(\begin{array}{cc}
T^{A} & 0 \\
0 & -\left(T^{A}\right)^{T}
\end{array}\right) .
$$

In the case of the simplest ansatz for the quark propagator, as in Eq. (14), the Schwinger-Dyson equation (34) reduces to an equation for the gap parameter $\Delta$. For this reason, it is also called the gap equation. After neglecting the dependence of the gap on the three-momentum and after performing the momentum integration, one arrives at the following approximate form of the gap equation in Euclidean space $\left(k_{4} \equiv i k_{0}\right)$ [28, 29, 30]:

$$
\Delta\left(k_{4}\right) \simeq \frac{\alpha_{s}}{9 \pi} \int \frac{d p_{4} \Delta\left(p_{4}\right)}{\sqrt{p_{4}^{2}+\Delta^{2}}} \ln \frac{\Lambda}{\left|k_{4}-p_{4}\right|},
$$

where $\Lambda=2(4 \pi)^{3 / 2} \mu \alpha_{s}^{-5 / 2}$. If the quark wave function renormalization from the diagonal part of the self-energy were taken into account, the extra factor $\exp \left(-\frac{4+\pi^{2}}{8}\right)$ would appear in the expression for $\Lambda$ [23]. The appearance of the logarithm in the integrand of the gap equation (36) is an artifact of the long-range force in QCD, mediated by the magnetic gluon modes. An approximate solution to the gap equation reads

$$
\Delta(0) \simeq \Lambda \exp \left(-\frac{3 \pi^{3 / 2}}{2^{3 / 2} \sqrt{\alpha_{s}}}\right)
$$


The result is plotted in Fig. 3, Here, the value of the coupling constant $\alpha_{s} \equiv \alpha_{s}(\mu)$ is taken at the scale of the quark chemical potential.

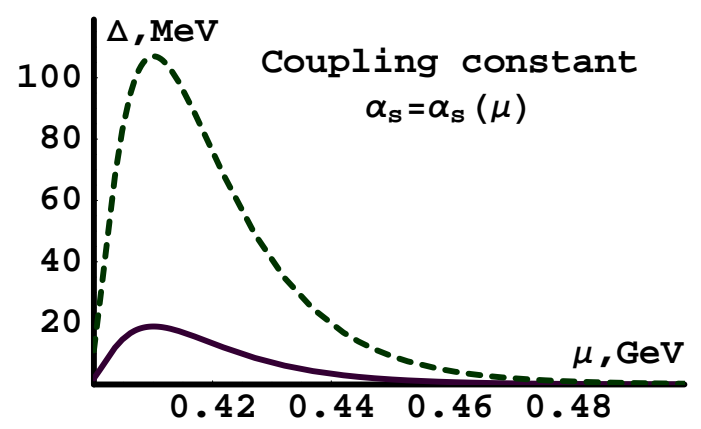

Figure 3: The value of the gap from Eq. (37) as a function of the quark chemical potential. The coupling constant is defined at the scale of the quark chemical potential. The solid (dashed) line gives the result with (without) taking the quark wave function renormalization into account.

It appears that, at densities that exist inside stars, i.e., $n \lesssim 10 n_{0}$, the value of the quark chemical potential cannot be much larger than $500 \mathrm{MeV}$. The corresponding value of the QCD coupling constant $\alpha_{s} \equiv \alpha_{s}(\mu)$ is not small, and, in contrast to the situation in asymptotically dense QCD, the Cooper pairing dynamics is not weakly interacting. This suggests that the above analysis of the Schwinger-Dyson equation may not be suitable for the description of matter inside stars. In spite of this, the solution in Eq. (37) is of fundamental importance in theory. This is one of very few non-perturbative solutions in QCD which is obtained from first principles, and which can be systematically improved by studying higher order corrections. Also, this solution shows that the phase diagram of QCD in the plane of temperature and chemical potential contains color superconducting phases at least at asymptotic densities.

\subsubsection{Gap equation in the NJL model}

If deconfined quark matter exists in the central regions of compact stars, it is likely to be color superconducting even when it is not weakly interacting. In order to study such a strongly interacting regime of quark matter with a typical value of the quark chemical potential $\mu \simeq 500 \mathrm{MeV}$, one can use 
various effective models of QCD. The simplest type of such models is the NJL model with a local four-fermion interaction in Eq. (9).

The NJL model in Eq. (9) lacks gluons, and, as reflection of this, it possesses the global instead of the local (gauge) $\mathrm{SU}(3)_{c}$ color symmetry. This model can be viewed as a result of integrating out heavy gluons from the QCD action. This is possible if the gluons get nonzero masses from nonperturbative effects. One arrives at an effective NJL model similar to that in Eq. (9) when the gauge fixing in QCD, needed to perform the integration, is consistent with the global color symmetry.

The global color symmetry of the NJL model is broken in the 2SC phase. Then, because of the Goldstone theorem, five massless Nambu-Goldstone bosons should appear in the low-energy spectrum of such a theory. In QCD, however, there is no room for such Nambu-Goldstone bosons. The seeming contradiction is removed by noting that these Nambu-Goldstone bosons are not physical. Their appearance is an artifact of the gauge fixing. In particular, there exist a gauge choice in QCD, namely the unitary gauge, in which these bosons can be completely eliminated.

The gap equation in the NJL model in the mean field approximation looks as follows:

$$
\Delta \simeq \frac{4 G_{D}}{\pi^{2}} \int_{0}^{\Lambda}\left(\frac{\Delta}{\sqrt{(p-\mu)^{2}+\Delta^{2}}}+\frac{\Delta}{\sqrt{(p+\mu)^{2}+\Delta^{2}}}\right) p^{2} d p .
$$

This gap equation can be obtained from a Schwinger-Dyson equation similar to that in QCD in Eq. (34) after the gluon long-range interaction is replaced by a local interaction proportional to $\delta(K-P)$.

The approximate solution to the gap equation in Eq. (38) reads

$$
\Delta \simeq 2 \sqrt{\Lambda^{2}-\mu^{2}} \exp \left(-\frac{\pi^{2}}{8 G_{D} \mu^{2}}+\frac{\Lambda^{2}-3 \mu^{2}}{2 \mu^{2}}\right) .
$$

This is very similar to the Bardeen-Cooper-Schrieffer solution in the case of low temperature superconductivity in solid state physics [9]. As in the Bardeen-Cooper-Schrieffer theory, it has the same type non-analytic dependence on the coupling constant and the same type dependence on the density of quasiparticle states at the Fermi surface.

When the quark chemical potential $\mu$ takes a value in the range between $400 \mathrm{MeV}$ and $500 \mathrm{MeV}$, and the strength of the diquark pairing is $G_{D}=\eta G_{S}$ with $\eta$ between 0.7 and 1 , the value of the gap appears to be of order $100 \mathrm{MeV}$. In essence, this is the result that was obtained in Ref. 13. 


\subsubsection{Properties of quark matter in the $2 \mathrm{SC}$ phase}

Now let us briefly summarize the main physical properties of the 2SC phase of dense quark matter. To large extent, these follow directly from the symmetry of the ground state, determined by the structure of the condensate in Eq. (10), and the magnitude of the diquark gap $\Delta$.

As we argued earlier, the blue up and blue down quarks do not participate in Cooper pairing in the $2 \mathrm{SC}$ phase. They give rise to gapless quasiparticles in the low energy spectrum of the theory. The density of states of such quasiparticles is proportional to $\mu^{2}$ and, therefore, is very large. This simple fact has important implications for the quark matter properties. At small temperatures, these gapless quasiparticles give dominant contributions to the specific heat, as well as to the electrical and heat conductivities of the 2SC phase. Also, the presence of the ungapped blue up and blue down quarks should result in a large neutrino emissivity due to the $\beta$-processes, $d_{b} \rightarrow u_{b}+e^{-}+\bar{\nu}_{e}$ and $u_{b}+e^{-} \rightarrow d_{b}+\nu_{e}$ 31.

The other four quark quasiparticles, originating from the red and green paired quarks, are gapped. Their dispersion relations look like that in Eq. (6). At small temperatures, $T \ll \Delta$, the contributions of these quasiparticles to all transport and many thermodynamic quantities are suppressed by the exponentially small factor $\exp (-\Delta / T)$. Gluons do not play a very important role either. They are bosons and their thermal number densities are small at small temperatures. In addition, five out of total eight gluons are gapped because of the color Meissner effect.

The presence of the unpaired blue quarks is also connected with the absence of baryon superfluidity in the $2 \mathrm{SC}$ phase. The generator of the baryon number conservation symmetry is defined as follows:

$$
\tilde{B}=B-\frac{2}{\sqrt{3}} T_{8}=\operatorname{diag}_{\text {color }}(0,0,1),
$$

where $B$ is the generator of the $\mathrm{U}(1)_{B}$ symmetry in vacuum. This vacuum generator $B$ mixes with the color generator $T_{8}$ to produce the generator $\tilde{B}$

of the $\tilde{\mathrm{U}}(1)_{B}$ symmetry in medium. From Eq. (401) it is clear that only (anti)blue quasiparticles carry a nonzero baryon number in the $2 \mathrm{SC}$ phase.

In the $2 \mathrm{SC}$ phase, there is also an unbroken $\tilde{\mathrm{U}}(1)_{\mathrm{em}}$ gauge symmetry. The corresponding generator reads

$$
\tilde{Q}=Q-\frac{1}{\sqrt{3}} T_{8},
$$


where $Q=\operatorname{diag}_{\text {flavor }}\left(\frac{2}{3},-\frac{1}{3}\right)$ is the generator of the $\mathrm{U}(1)$ em symmetry in vacuum. The gauge boson of $\tilde{U}(1)_{\mathrm{em}}$ is nothing else but the medium photon. It should be clear, therefore, that the 2SC ground state is not subject to the electromagnetic Meissner effect. Thus, if there is an external magnetic field, it is not expelled from such a medium.

One should notice that the quark quasiparticles carry the following $\tilde{Q}_{-}$ charges:

$$
\begin{array}{cc}
\tilde{Q}\left(u_{r}\right)=\tilde{Q}\left(u_{g}\right)=\frac{1}{2}, & \tilde{Q}\left(u_{b}\right)=1, \\
\tilde{Q}\left(d_{r}\right)=\tilde{Q}\left(d_{g}\right)=-\frac{1}{2}, & \tilde{Q}\left(d_{b}\right)=0 .
\end{array}
$$

It is interesting that the blue up quark, which remains unpaired in the 2SC phase, is charged (this is not true for the blue down quark which is also unpaired). It means that this phase of matter is a $\tilde{Q}$-conductor, and that the value of the electrical conductivity is very high.

The pressure of cold dense quark matter is dominated by the Pauli pressure. The partial contribution of each quark is $\mu^{4} / 12 \pi^{2}$, see Eq. (2). The correction due to color superconductivity is parametrically suppressed by the factor $(\Delta / \mu)^{2}$. The reason is that the Cooper pairing affects only those quark states that are close to the Fermi surface. In contrast, it is the whole Fermi sphere that contributes to the Pauli pressure. At weak coupling, the contribution due to diquark pairing can be easily calculated. It is given by $(\mu \Delta / 2 \pi)^{2}$ per each gapped quark quasiparticle 32. In the 2SC phase, there are six (two flavors times three colors) quarks in total, and four of them give rise to quasiparticles with the gap $\Delta$ in their energy spectra. Thus, the pressure of such matter is approximately given by [32, 33.

$$
P_{(2 \mathrm{SC})} \simeq \frac{N_{c} N_{f} \mu^{4}}{12 \pi^{2}}-B+4\left(\frac{\mu \Delta}{2 \pi}\right)^{2}=\frac{\mu^{4}}{2 \pi^{2}}-B+\frac{\mu^{2} \Delta^{2}}{\pi^{2}} .
$$

Note that the bag pressure was also added on the right hand side. If present, this term partially cancels the leading $\mu^{4}$ term. Because of this, color superconductivity may have a large effect on the hadron-quark phase transition [34] and even on the properties of compact stars 35]. By making use of thermodynamic identities, one can derive the corresponding energy density,

$$
\epsilon_{(2 \mathrm{SC})} \simeq \frac{3 \mu^{4}}{2 \pi^{2}}+B+\frac{\mu^{2} \Delta^{2}}{\pi^{2}}\left(1+\frac{2 \mu}{\Delta} \frac{\partial \Delta}{\partial \mu}\right) .
$$


The expressions in Eqs. (44) and (45) give a parametric representation of the equation of state of dense quark matter in the 2SC phase. Such an equation of state is the key input in the Tolman-Oppenheimer-Volkoff equations [36] which determine the interior structure of compact stars. In short, such equations describe hydrostatic equilibrium of matter inside a star when the pressure gradient in each radial layer of the star is balanced by the gravitational weight of the layer itself. Several constructions of compact stars with quark matter in their interior were presented in Refs. [34, 35, 37, 38, 39, 40, 41.

\subsection{Color-flavor locked phase $\left(N_{f}=3\right)$}

It may happen that dense baryonic matter is made not only of the lightest up and down quarks, but of strange quarks as well. In fact, because of a possible reduction in the free energy from converting non-strange quarks into strange quarks, one may even speculate that strange quark matter is the true ground state of baryonic matter 42 .

The constituent strange quark mass in vacuum QCD is estimated to be of order $500 \mathrm{MeV}$. Its current mass is about $100 \mathrm{MeV}$. In dense baryonic matter (say, with $\mu \simeq 500 \mathrm{MeV}$ ), the value of the strange quark mass should be in the range between $100 \mathrm{MeV}$ and $500 \mathrm{MeV}$. It is plausible then that strange quarks also participate in Cooper pairing.

Here, we consider an idealized version of three-flavor quark matter, in which all quarks are assumed to be massless. The more realistic case of a nonzero strange quark mass will be briefly discussed in the second lecture. In the massless case, the quark model possesses the global $\mathrm{SU}(3)_{L} \times \mathrm{SU}(3)_{R}$ chiral symmetry and the global $\mathrm{U}(1)_{B}$ symmetry connected with the baryon number conservation. This is in addition to $\mathrm{SU}(3)_{c}$ color gauge symmetry. Note that the generator $Q=\operatorname{diag}_{\text {flavor }}\left(\frac{2}{3},-\frac{1}{3},-\frac{1}{3}\right)$ of the $\mathrm{U}(1)_{\mathrm{em}}$ symmetry of electromagnetism is traceless, and therefore it coincides with one of the vector-like generators of the $\mathrm{SU}(3)_{L} \times \mathrm{SU}(3)_{R}$ chiral group.

To large extent, the color and flavor structure of the diquark condensate of Cooper pairs in the three-flavor quark matter is again determined by the symmetry of the attractive diquark channel (i.e., color-antisymmetric antitriplet) and the Pauli exclusion principle. The spin-0 condensate corresponds to the following ground state expectation value [43]:

$$
\left\langle\left(\bar{\psi}^{C}\right)_{i}^{a} \gamma^{5} \psi_{j}^{b}\right\rangle \sim \sum_{I, J=1}^{3} c_{J}^{I} \varepsilon_{i j I} \epsilon^{a b J}+\cdots
$$


which is antisymmetric in the color and flavor indices of the constituent quarks, cf. Eq. (10). The $3 \times 3$ matrix $c_{J}^{I}$ is determined from the global minimum of the free energy. It appears that $c_{J}^{I}=\delta_{J}^{I}$. The ellipsis on the right hand side stand for a contribution which is symmetric in color and flavor. A small contribution of this type is always induced in the ground state, despite the fact that it corresponds to a repulsive diquark channel 43, 44. This is not surprising after noting that the symmetric condensate $\left\langle\left(\bar{\psi}^{C}\right)_{i}^{a} \gamma^{5} \psi_{j}^{b}\right\rangle \sim \delta_{i}^{a} \delta_{j}^{b}+\delta_{j}^{a} \delta_{i}^{b}$ does not break any additional symmetries [43].

In the ground state, determined by the condensate (46), the chiral symmetry is broken down to its vector-like subgroup. However, the mechanism of this symmetry breaking is very unusual. To see this, let us rewrite the condensate in Eq. (46) as follows:

$$
\left\langle\psi_{L, i}^{a, \alpha} \epsilon_{\alpha \beta} \psi_{L, j}^{b, \beta}\right\rangle=-\left\langle\psi_{R, i}^{a, \dot{\alpha}} \epsilon_{\dot{\alpha} \dot{\beta}} \psi_{R, j}^{b, \dot{\beta}}\right\rangle \sim \sum_{I=1}^{3} \varepsilon_{i j I} \epsilon^{a b I}+\cdots,
$$

where $\alpha, \beta, \dot{\alpha}, \dot{\beta}=1,2$ are the spinor indices. The condensate of left-handed fields in Eq. (47) breaks the $\mathrm{SU}(3)_{c}$ color symmetry and the $\mathrm{SU}(3)_{L}$ chiral symmetry, but leaves the diagonal $\mathrm{SU}(3)_{L+c}$ subgroup unbroken. Indeed, as one can check, this condensate remains invariant under the simultaneous flavor transformation $g_{L}$ and the compensating color transformation $g_{c}=$ $\left(g_{L}\right)^{-1}$. Similarly, the condensate of right-handed fields in Eq. (47) leaves the $\mathrm{SU}(3)_{R+c}$ subgroup unbroken.

When both condensates are present, the symmetry of the ground state is $\mathrm{SU}(3)_{L+R+c}$. At the level of global symmetries, the original $\mathrm{SU}(3)_{L} \times \mathrm{SU}(3)_{R}$ is broken down to the vector-like $\mathrm{SU}(3)_{L+R}$, just like in vacuum. Unlike in vacuum, however, this breaking does not result from any condensates mixing left- and right-handed fields. Instead, it results from two separate condensates, made of left-handed fields only and of right-handed fields only. The color-flavor orientations of the two condensates are "locked" to each other by color transformations. This mechanism is called locking, and the corresponding phase of matter is called color-flavor-locked (CFL) phase [43].

To large extent, the gap equation in the three-flavor quark matter is the same as in the two-flavor case. The differences come only from a slightly more complicated color-flavor structure of the off-diagonal part of the inverse quark propagator (gap matrix) [43, 44,

$$
\Delta_{a b}^{i j}=i \gamma^{5}\left[\frac{1}{3}\left(\Delta_{1}+\Delta_{2}\right) \delta_{a}^{i} \delta_{b}^{j}-\Delta_{2} \delta_{b}^{i} \delta_{a}^{j}\right],
$$


where two parameters $\Delta_{1}$ and $\Delta_{2}$ determine the values of the gaps in the quasiparticles spectra. In the ground state that respects the $\mathrm{SU}(3)_{L+R+c}$ symmetry, the original nine quark states give rise to a singlet and an octet of quasiparticles. The singlet has gap $\Delta_{1}$, and the octet has gap $\Delta_{2}$.

When the small symmetric diquark condensate is neglected, one finds that $\Delta_{1}=2 \Delta_{2}$, i.e., the gap of the singlet is twice as large as the gap of the octet.

In real case, however, this relation holds only approximately. The value of the octet gap $\Delta_{2}$ in asymptotically dense QCD is given by an expression similar to that in Eq. (37), but the overall coefficient is multiplied by an extra factor $2^{-1 / 3}(2 / 3)^{5 / 2} \approx 0.288[44,45$.

\subsubsection{Properties of quark matter in the CFL phase}

Let us briefly review physical properties of the CFL phase. In contrast to the $2 \mathrm{SC}$ phase, there are no gapless quark quasiparticles in the low energy spectrum of the CFL phase. This implies that, at small temperatures, $T \ll \Delta$, the contributions of quark quasiparticles to all transport and many thermodynamic quantities are suppressed by the exponentially small factor $\exp (-\Delta / T)$. Gluons do not play any important role either. All of them are gapped because of the color Meissner effect.

Unlike the 2SC phase, the CFL phase is superfluid. This is because the $\mathrm{U}(1)_{B}$ baryon number symmetry is broken in the ground state. If such a phase appears in a core of a rotating star, it will be threaded with rotational vortices. This may be related to the existence of such phenomena as glitches. These are sudden changes of the rotational frequency observed in some pulsars. The glitches may be caused by occasional releasing of the angular momentum of rotational vortices, that remained pinned to the stellar crust [46].

Because of the Goldstone theorem, the breaking of the $\mathrm{U}(1)_{B}$ baryon number symmetry should result in the appearance of a Nambu-Goldstone boson in the low-energy theory. In absence of gapless quark quasiparticles, this Nambu-Goldstone boson turns out to play an important role in many transport properties of cold CFL matter [33, 47].

The CFL phase, like the 2SC phase, is not an electromagnetic superconductor. Therefore, it does not expel a magnetic flux from its interior. This is the result of having an unbroken $\tilde{\mathrm{U}}(1)_{\mathrm{em}}$ gauge symmetry in the ground 
state. The corresponding generator reads

$$
\tilde{Q}=Q-T_{3}-\frac{1}{\sqrt{3}} T_{8} .
$$

The quark quasiparticles carry the following $\tilde{Q}$-charges:

$$
\begin{aligned}
\tilde{Q}\left(u_{r}\right)=0, & \tilde{Q}\left(u_{g}\right)=\tilde{Q}\left(u_{b}\right)=1, \\
\tilde{Q}\left(d_{r}\right)=-1, & \tilde{Q}\left(d_{g}\right)=\tilde{Q}\left(d_{b}\right)=0, \\
\tilde{Q}\left(s_{r}\right)=-1, & \tilde{Q}\left(s_{g}\right)=\tilde{Q}\left(s_{b}\right)=0 .
\end{aligned}
$$

Since all quasiparticles are gapped, the corresponding phase of matter is a $\tilde{Q}$-insulator. Its electrical conductivity at small temperatures is dominated by thermally excited electrons and positrons [47]. At sufficiently low temperatures, when the thermal density is low, the CFL phase becomes transparent to light [47, 48.

In order to write down the expression for the pressure of three-flavor quark matter in the CFL phase, we take the Pauli pressure contributions of nine (three flavors times three colors) quarks and add the correction due to color superconductivity from one quasiparticle with gap $\Delta_{1}$ and eight quasiparticles with gap $\Delta_{2}$. Thus, we derive

$$
\begin{aligned}
P_{(\mathrm{CFL})} & \simeq \frac{N_{c} N_{f} \mu^{4}}{12 \pi^{2}}-B+\left(\frac{\mu \Delta_{1}}{2 \pi}\right)^{2}+8\left(\frac{\mu \Delta_{2}}{2 \pi}\right)^{2} \\
& \simeq \frac{3 \mu^{4}}{4 \pi^{2}}-B+3 \frac{\mu^{2} \Delta^{2}}{\pi^{2}},
\end{aligned}
$$

where we used the approximate relation between the singlet and octet gaps, $\Delta_{1}=2 \Delta_{2} \equiv 2 \Delta$. By making use of thermodynamic identities, one can derive the corresponding energy density,

$$
\epsilon_{(\mathrm{CFL})} \simeq \frac{9 \mu^{4}}{4 \pi^{2}}+B+3 \frac{\mu^{2} \Delta^{2}}{\pi^{2}}\left(1+\frac{2 \mu}{\Delta} \frac{\partial \Delta}{\partial \mu}\right) .
$$

These two expressions give a parametric representation of the equation of state of dense quark matter in the CFL phase.

\subsubsection{Low-energy effective action}

As in the case of chiral perturbation theory in vacuum QCD [49], one could write down an effective low-energy theory in the CFL phase. From the symmetry breaking pattern, it is known that there are nine Nambu-Goldstone 
bosons and one pseudo-Nambu-Goldstone boson in the low-energy spectrum of the theory [50, 51. Eight of the Nambu-Goldstone bosons are similar to those in vacuum QCD: three pions $\left(\pi^{0}\right.$ and $\left.\pi^{ \pm}\right)$, four kaons $\left(K^{0}, \bar{K}^{0}\right.$ and $K^{ \pm}$) and the eta-meson $(\eta)$. The additional Nambu-Goldstone boson $(\phi)$ comes from breaking the $\mathrm{U}(1)_{B}$ baryon symmetry. Finally, the pseudoNambu-Goldstone boson $\left(\eta^{\prime}\right)$ results from breaking the approximate axial $\mathrm{U}(1)_{A}$ symmetry.

The low energy action for the Nambu-Goldstone bosons in the CFL phase was derived in Refs. 50, 51, 52. In the chiral limit, the result reads

$$
\begin{aligned}
\mathcal{L}_{\text {eff }} & =\frac{f_{\pi}^{2}}{4} \operatorname{Tr}\left[\partial_{0} \Sigma \partial_{0} \Sigma^{\dagger}-v^{2} \partial_{i} \Sigma \partial_{i} \Sigma^{\dagger}\right]+\frac{1}{2}\left[\left(\partial_{0} \phi\right)^{2}-v^{2}\left(\partial_{i} \phi\right)^{2}\right] \\
& +\frac{1}{2}\left[\left(\partial_{0} \eta^{\prime}\right)^{2}-v^{2}\left(\partial_{i} \eta^{\prime}\right)^{2}\right]
\end{aligned}
$$

where $f_{\pi}^{2}=(21-8 \ln 2)(\mu / 6 \pi)^{2}$ and $v^{2}=1 / 3$ were calculated in asymptotically dense QCD in Refs. [52, 53. (A nonzero mass of the $\eta^{\prime}$-meson was neglected here [54].) By definition, $\Sigma \equiv \exp \left(i \lambda^{A} \pi^{A} / f_{\pi}\right)$ is a unitary matrix field which describes the octet of the Nambu-Goldstone bosons, transforming under the chiral $S U(3)_{L} \times S U(3)_{R}$ group as follows:

$$
\Sigma \rightarrow U_{L} \Sigma U_{R}^{\dagger}
$$

where $\left(U_{L}, U_{R}\right) \in S U(3)_{L} \times S U(3)_{R}$.

The extra terms in the low-energy action should be added when quark masses are nonzero [52, 55]. Since the quark masses break the chiral symmetry of dense QCD explicitly, these type of corrections produce non-vanishing masses for all Nambu-Goldstone bosons, except one. The single boson left massless is the Nambu-Goldstone boson related to the baryon number breaking. Of course, this symmetry is not affected by the quark masses.

Within the framework of the high density effective theory [45, 56, it was shown that an additional effect of nonzero quark masses may appear when the strange quark mass exceeds a critical value $m_{s} \sim m_{u}^{1 / 3} \Delta^{2 / 3}$. In this case, the CFL phase is expected to undergo a phase transition to a phase with a meson (e.g., kaon or/and eta) condensate [57, 58, 59]. In the low-energy action of the corresponding phase, additional Nambu-Goldstone bosons with very unusual properties can appear 60]. 


\subsection{Spin-1 color superconductivity $\left(N_{f}=1\right)$}

In the case of neutral matter in $\beta$ equilibrium, as we shall discuss in the second lecture in more detail, it may happen that different quark flavors cannot create Cooper pairs because of a large mismatch between their Fermi momenta. In this case, one could consider the possibility of a much weaker spin-1 Cooper pairing [12, 30, 61, 62, 63, 64]. It is the Pauli principle that does not allow to construct spin-0 Cooper pairs from quarks of the same flavor. The color antisymmetric wave function of a pair can only be symmetric in spin indices. This corresponds to a spin-1 state.

The pairing in a spin-1 triplet state and in a color antisymmetric antitriplet state can lead to a rather complicated structure of the diquark condensate. The general structure of the gap matrix can be written in the following form [64]:

$$
\Delta^{a b}=i \Delta_{0} \sum_{c, i=1}^{3} \epsilon^{a b c} \mathcal{C}_{c i}\left[\hat{k}^{i} \cos \theta+\gamma_{\perp}^{i} \sin \theta\right],
$$

where $\hat{\mathbf{k}} \equiv \mathbf{k} / k, \gamma_{\perp}^{i} \equiv \gamma^{i}-\hat{k}^{i}(\boldsymbol{\gamma} \cdot \hat{\mathbf{k}})$, and the explicit form of the $3 \times 3$ matrix $\mathcal{C}_{c i}$ as well as the angular parameter $\theta$ are determined by the minimization of the quark matter free energy. In the special cases with $\theta=0$ and $\theta=\pi / 2$, the corresponding gaps are called longitudinal and transverse, respectively.

By choosing various specific matrices $\mathcal{C}$, one could construct many different spin-1 phases. The four most popular of them are the so-called A-phase, the color-spin-locked (CSL) phase, the polar and the planar phases. These are determined by the following matrices [64]:

$$
\begin{aligned}
\mathcal{C}^{(\mathrm{A}-\text { phase })}=\frac{1}{\sqrt{2}}\left(\begin{array}{ccc}
0 & 0 & 0 \\
0 & 0 & 0 \\
1 & i & 0
\end{array}\right), & \mathcal{C}^{(\mathrm{CSL})}=\frac{1}{\sqrt{3}}\left(\begin{array}{ccc}
1 & 0 & 0 \\
0 & 1 & 0 \\
0 & 0 & 1
\end{array}\right) \\
\mathcal{C}^{\text {(polar) }}=\left(\begin{array}{lll}
0 & 0 & 0 \\
0 & 0 & 0 \\
0 & 0 & 1
\end{array}\right), & \mathcal{C}^{(\text {planar })}=\frac{1}{\sqrt{2}}\left(\begin{array}{ccc}
1 & 0 & 0 \\
0 & 1 & 0 \\
0 & 0 & 0
\end{array}\right) .
\end{aligned}
$$

The corresponding four phases are characterized by different symmetries of their ground state. In particular, the original group $\mathrm{SU}(3)_{c} \times \mathrm{SO}(3)_{J} \times \mathrm{U}(1)_{\mathrm{em}}$ of one-flavor quark matter breaks down as follows [62, 63, 64]:

$$
\text { A-phase: } \quad S U(2)_{c} \times \widetilde{S O}(2)_{J} \times \tilde{U}(1)_{\mathrm{em}},
$$




$$
\begin{aligned}
\text { CSL: } & \widetilde{S O}(3)_{J}, \\
\text { Polar: } & S U(2)_{c} \times S O(2)_{J} \times \tilde{U}(1)_{\mathrm{em}}, \\
\text { Planar: } & \widetilde{S O}(2)_{J} \times \tilde{U}(1)_{\mathrm{em}} .
\end{aligned}
$$

It was argued recently that the phase with the lowest free energy is the transverse A-phase 64.

The value of the spin-1 gap is estimated to be about two or three orders of magnitude smaller than a typical spin-0 gap. At best, it can be about $1 \mathrm{MeV}$, but more realistically it is expected to be about $0.1 \mathrm{MeV}$, or less. The presence of such a small gap is unlikely to modify considerably any thermodynamic, or even transport properties of dense quark matter. It is fair to mention, however, that the cooling history of stars might be sensitive enough to feel the effect of such small gaps [65]. It was also suggested that the electromagnetic properties of spin-1 phases can be of phenomenological importance 63]. In contrast to the $2 \mathrm{SC}$ and CFL phases, discussed earlier, spin-1 phases reveal the electromagnetic Meissner effect. As one can see from Eq. (61), this clearly applies to the CSL phase. It turns out that the electromagnetism is also subject to the Meissner effect when there appears a mixture of any two independent spin- 1 condensates made of quarks with different charges (e.g., one is made of up quarks and the other is made of down quarks) 63. The type-I superconductivity in such a system could have observable effects on the magnetic field relaxation in pulsars.

\subsection{Summary of the first lecture}

In this lecture, only the main three color superconducting phases of dense quark matter have been discussed. These are the simplest possible phases that can be realized in one-, two-, and three-flavor quark matter. It should be emphasized, however, that the conditions in dense matter were idealized by assuming that the chemical potentials of pairing quarks were equal. In real situation which, for example, is realized in the interior of compact stars, matter happens to be very different from ideal. In particular, the charge neutrality and the $\beta$ equilibrium are two very important conditions that may modify the quark chemical potentials and, therefore, affect the properties of quark matter. These issues are discussed in the second lecture.

The aim of this lecture was to introduce the general idea of color superconductivity in cold dense baryonic matter. It was argued that such matter

is expected to be deconfined and, because of the Cooper instability, it should 
be color superconducting. To large extent, this phenomenon is the same as low-temperature superconductivity in condensed matter physics [9]. One feature in dense QCD is very remarkable. The attractive interaction between quarks comes from the gauge boson exchange. This is in contrast to the Bardeen-Cooper-Schrieffer theory in solid state physics, where the gauge boson (photon) exchange is repulsive, while an attractive interaction comes from the phonon exchange.

One of the key points of this lecture is the observation that QCD at asymptotic densities is a weakly interacting (although non-perturbative) theory. Moreover, the properties of its color superconducting ground state can be studied analytically from first principles, providing a rare example of an essentially solvable limit in a non-Abelian theory. By itself, this has a fundamental theoretical importance. Also, this result may provide valuable insights in the theory of strong interactions. One of the examples might be the idea of duality between the hadronic and quark description of QCD [66].

Although the use of weakly interacting QCD for the description of dense quark matter is very instructive by itself, it does not seem to be very useful for the quantitative description of the ground state at densities existing inside stars. The reason is that the density of matter in stars is bounded from above by the condition of the hydrostatic equilibrium, $n \lesssim 10 n_{0}$. At such not so large densities, one cannot treat QCD as a weakly interacting theory. In this situation, the use of various effective models of QCD has proved to be very useful. 


\section{Color superconductivity in neutral matter}

\subsection{Dense matter inside stars}

As we discussed in the first lecture, it is natural to expect that some color superconducting phases may exist in the interior of compact stars. The estimated central densities of such stars might be sufficiently large for producing deconfined quark matter. Such matter should develop the Cooper instability and become color superconducting. It should also be noted that typical

temperatures inside compact stars are so low that the diquark condensate, if produced, would not melt.

In the first lecture, we discussed the idealized version of dense matter, in which the Fermi momenta of pairing quarks were assumed to be equal. This does not describe the real situation that should occur inside compact stars. The reason is that matter in the bulk of a compact star should be neutral (at least, on average) with respect to electric as well as color charges. Otherwise, the star would not be bound by gravity which is much weaker than electromagnetism. Matter should also remain in $\beta$ equilibrium, i.e., all $\beta$ processes (e.g., such as $d \rightarrow u+e^{-}+\bar{\nu}_{e}, u+e^{-} \rightarrow d+\nu_{e}, s \rightarrow u+e^{-}+\bar{\nu}_{e}$, and $\left.u+e^{-} \rightarrow s+\nu_{e}\right)$ should go with equal rates in both directions.

As we shall see below, after the charge neutrality and the $\beta$ equilibrium in quark matter are enforced, the chemical potentials of different quarks would satisfy some relations that may interfere with the dynamics of Cooper pairing. If this happens, some color superconducting phases may become less favored than others. For example, it was argued in Ref. 67, that a mixture of unpaired strange quarks and the non-strange 2SC phase, made of up and down quarks, is less favorable than the CFL phase after the charge neutrality condition is enforced. A similar conclusion was also reached in Ref. 68].

Assuming that the constituent medium modified mass of the strange quark is large (i.e., larger than the corresponding strange quark chemical potential), in Ref. 69 it was shown that neutral two-flavor quark matter in $\beta$-equilibrium can have another rather unusual ground state called the gapless two-flavor color superconductor (g2SC). While the symmetry in the g2SC ground state is the same as that in the conventional 2SC phase, the spectrum of the fermionic quasiparticles is different.

The existence of gapless color superconducting phases was confirmed in Refs. [70, 171, 72, and generalized to nonzero temperatures in Refs. [73, 74]. In addition, it was also shown that a gapless CFL (gCFL) phase could appear 
in neutral strange quark matter [75, 76]. At nonzero temperature, the gCFL phase and several other new phases (e.g., the so-called dSC and uSC phases) were studied in Refs. [77, 78, 79]. If the surface tension is sufficiently small, as suggested in Ref. [80, the mixed phase composed of the 2SC phase and the normal quark phase 39 may be more favored.

Here, it may be appropriate to mention that a non-relativistic analogue of gapless superconducting phases could appear in a trapped gas of cold fermionic atoms [81, 82, 83, 84, 85. (Note that an alternative ground state for the atomic system, similar to the quark mixed phases in Refs. [80, 39, 86], was proposed in Ref. [87.)

\subsection{Gapless two-flavor color superconductivity}

\subsubsection{Neutrality vs. color superconductivity}

Let us discuss the effect of charge neutrality on color superconductivity. It is instructive to start with the case of two-flavor quark matter first. In this case, the effect is most prominent and, thus, it is easiest to understand. Later, the results for three-flavor quark matter will be also discussed.

In order to get an impression about the importance of the charge neutrality condition in a large macroscopic chunk of matter, such as a core of a compact star, let us estimate the corresponding Coulomb energy. A simple calculation leads to the following result:

$$
E_{\text {Coulomb }} \sim n_{Q}^{2} R^{5} \sim M_{\odot} c^{2}\left(\frac{n_{Q}}{10^{-15} e / \mathrm{fm}^{3}}\right)^{2}\left(\frac{R}{1 \mathrm{~km}}\right)^{5},
$$

where $R$ is the radius of the quark matter core, whose charge density is denoted by $n_{Q}$. It is easy to see that this energy is not an extensive quantity: the value of the corresponding energy density increases with the size of the system as $R^{2}$. By taking a typical value of the charge density in the ideal 2SC phase, $n_{Q} \sim 10^{-2} e / \mathrm{fm}^{3}$, the energy in Eq. (64) becomes a factor of $10^{26}$ larger than the rest mass energy of the Sun! To avoid such an incredibly large energy price, the charge neutrality $n_{Q}=0$ should be satisfied with a very high precision.

In the case of two-flavor quark matter, one can argue that the neutrality is achieved if the number density of down quarks is approximately twice as large as number density of up quarks, $n_{d} \approx 2 n_{u}$. This follows from the fact that the negative charge of the down quark $\left(Q_{d}=-1 / 3\right)$ is twice as small as the 
positive charge of the up quark $\left(Q_{u}=2 / 3\right)$. When $n_{d} \approx 2 n_{u}$, the total electric charge density is vanishing in absence of electrons, $n_{Q} \approx Q_{d} n_{d}+Q_{u} n_{u} \approx 0$. It turns out that even a nonzero density of electrons, required by the $\beta$ equilibrium condition, could not change this relation much.

The argument goes as follows. Let us consider noninteracting massless quarks. In $\beta$ equilibrium, the chemical potentials of the up quark and the down quark, $\mu_{u}$ and $\mu_{d}$, should satisfy the relation $\mu_{d}=\mu_{u}+\mu_{e}$ where $\mu_{e}$ is the chemical potential of electrons (i.e., up to a sign, the chemical potential of the electric charge). By assuming that $\mu_{d} \approx 2^{1 / 3} \mu_{u}$, i.e., $n_{d} \approx 2 n_{u}$ as required by the neutrality in absence of electrons, one obtains the following result for

the electron chemical potential: $\mu_{e}=\mu_{d}-\mu_{u} \approx \frac{1}{4} \mu_{u}$. The corresponding density of electrons is $n_{e} \approx 5 \cdot 10^{-3} n_{u}$. We see that $n_{e} \ll n_{u}$ which is in agreement with the original assumption that $n_{d} \approx 2 n_{u}$ in neutral matter.

While the approximate relation $n_{d} \approx 2 n_{u}$ may be slightly modified in an interacting system, the main conclusion would remain qualitatively the same. The Fermi momenta of up and down quarks, whose pairing is responsible for color superconductivity, are generally non-equal after the neutrality and $\beta$ equilibrium conditions are imposed. In this case, the Cooper pairing may be substantially modified, or even prevented. The study of this issue is the topic of the rest of this lecture.

\subsubsection{Model}

In view of strongly coupled dynamics and various non-perturbative (e.g., instanton) effects in dense QCD at moderate densities $n \lesssim 10 n_{0}$, existing in central regions of compact stars, there is no real advantage in using microscopic theory of QCD. For purposes of the current presentation, it suffices to use an effective model such, for example, as the NJL model with the Lagrangian density in Eq. (9), in which the common quark chemical potential $\mu$ is replaced by a properly chosen color-flavor matrix $\hat{\mu}$ of chemical potentials 67. 68, 17.

In $\beta$ (chemical) equilibrium, the matrix $\hat{\mu}$ is determined only by a few independent chemical potentials which can be introduced in the corresponding partition function as Lagrange multipliers in front of conserved charges $Q_{i}$ of the model,

$$
Z=\operatorname{Tr} \exp \left(-\frac{H+\sum_{i} \mu_{i} Q_{i}}{T}\right)
$$

In two-flavor quark matter, we consider only three relevant conserved charges: 
the baryon number $n_{B}$, the electric charge $n_{Q}$, and the color charge $n_{8}$. Then, the matrix of quark chemical potentials is given in terms of the baryon chemical potential (by definition, $\mu_{B} \equiv 3 \mu$ ), the electron chemical potential $\left(\mu_{e}\right)$ and the color chemical potential $\left(\mu_{8}\right)$ [69,

$$
\hat{\mu}_{i j, \alpha \beta}=\left(\mu \delta_{i j}-\mu_{e} Q_{i j}\right) \delta_{\alpha \beta}+\frac{2}{\sqrt{3}} \mu_{8} \delta_{i j}\left(T_{8}\right)_{\alpha \beta}
$$

where $Q$ and $T_{8}$ are the generators of $\mathrm{U}(1)_{\mathrm{em}}$ of electromagnetism and the $\mathrm{U}(1)_{8}$ subgroup of $\mathrm{SU}(3)_{c}$ color gauge group. The explicit expressions for chemical potentials of different quarks read

$$
\begin{aligned}
\mu_{u r}=\mu_{u g} & =\mu-\frac{2}{3} \mu_{e}+\frac{1}{3} \mu_{8}, \\
\mu_{d r}=\mu_{d g} & =\mu+\frac{1}{3} \mu_{e}+\frac{1}{3} \mu_{8}, \\
\mu_{u b} & =\mu-\frac{2}{3} \mu_{e}-\frac{2}{3} \mu_{8}, \\
\mu_{d b} & =\mu+\frac{1}{3} \mu_{e}-\frac{2}{3} \mu_{8} .
\end{aligned}
$$

Note that there exist two mutually commuting color charges in the model. They are related to the generators $T_{3}$ and $T_{8}$ of the $\mathrm{SU}(3)_{c}$ gauge group. Then, generally speaking, one could introduce two independent chemical potentials for the corresponding two color charges. Because of the invariance of the 2SC ground state under the transformations of the $\mathrm{SU}(2)_{c}$ color gauge subgroup, the introduction of the second nontrivial color chemical potential $\mu_{3}$ is not necessary. This additional color chemical potential, however, is generally needed in three-flavor quark matter.

Here, several comments are in order regarding the introduction of color quark chemical potential $\mu_{8}$. This is required for enforcing color charge neutrality only in the color superconducting ground state. In the NJL model, it is added by hand in the partition function, and then adjusted to neutralize the ground state. In QCD, on the other hand, the charge neutrality is realized dynamically due to the generation of the gluon condensate $\left\langle A_{0}^{8}\right\rangle \neq 0[88$. The appearance of such a condensate is equivalent to having a nonzero value of the chemical potential $\mu_{8} \sim g_{s}\left\langle A_{0}^{8}\right\rangle$. A similar relation exists between the chemical potential $\mu_{3}$ and the gluon condensate $\left\langle A_{0}^{3}\right\rangle$ in three-flavor quark matter. It should be clear, therefore, that nonzero gluon condensates, such as $\left\langle A_{0}^{8}\right\rangle$ or $\left\langle A_{0}^{3}\right\rangle$, do not break explicitly any symmetries in QCD. They appear 
spontaneously only in the color superconducting ground state, and only after gauge fixing.

\subsubsection{Effective potential}

In order to derive the effective potential in the NJL model (9) in the mean field approximation, it is convenient to use the standard Hubbard-Stratonovich method 89. Then, the original NJL Lagrangian density is replaced by

$$
\begin{aligned}
\tilde{\mathcal{L}}_{\mathrm{NJL}} & =\bar{\psi}\left[i \gamma^{\mu} \partial_{\mu}+\gamma^{0} \hat{\mu}-m^{(0)}-\sigma-i \gamma_{5}(\overrightarrow{\boldsymbol{\pi}} \cdot \vec{\tau})\right] \psi-\frac{\sigma^{2}+\overrightarrow{\boldsymbol{\pi}}^{2}}{4 G_{S}} \\
& -\frac{\phi_{a}^{*} \phi^{a}}{4 G_{D}}-\frac{i}{2} \bar{\psi}^{C} \gamma_{5} \varepsilon \epsilon^{a} \phi^{a} \psi-\frac{i}{2} \bar{\psi} \varepsilon \epsilon^{a} \phi_{a}^{*} \gamma_{5} \psi^{C}
\end{aligned}
$$

where $\sigma, \vec{\pi}$, and $\phi_{a}$ are auxiliary fields. After taking into account the equations of motion for these fields,

$$
\begin{aligned}
\sigma & =-2 G_{S}(\bar{\psi} \psi), \\
\overrightarrow{\boldsymbol{\pi}} & =-2 G_{S}\left(\bar{\psi} i \gamma_{5} \vec{\tau} \psi\right), \\
\phi^{a} & =-2 G_{D}\left(i \bar{\psi} \varepsilon \epsilon^{a} \gamma_{5} \psi^{C}\right), \\
\phi_{a}^{*} & =-2 G_{D}\left(i \bar{\psi}^{C} \gamma_{5} \varepsilon \epsilon^{a} \psi\right),
\end{aligned}
$$

one can check that the Lagrangian density in (171) is equivalent to the original NJL model. In the mean field approximation, the auxiliary fields are replaced by their vacuum expectation values, $\langle\sigma\rangle=m-m^{(0)},\langle\overrightarrow{\boldsymbol{\pi}}\rangle=0$ and $\left\langle\phi^{a}\right\rangle=\Delta \delta_{3}^{a}$,

and the quantum fluctuations are neglected. Then, the effective potential of quark matter in $\beta$-equilibrium (with massless electrons) takes the form [73:

$$
\begin{aligned}
\Omega & =\Omega_{0}-\frac{1}{12 \pi^{2}}\left(\mu_{e}^{4}+2 \pi^{2} T^{2} \mu_{e}^{2}+\frac{7 \pi^{4}}{15} T^{4}\right)+\frac{\left(m-m^{(0)}\right)^{2}}{4 G_{S}} \\
& +\frac{\Delta^{2}}{4 G_{D}}-\sum_{I} \int \frac{d^{3} p}{(2 \pi)^{3}}\left[E_{I}+2 T \ln \left(1+e^{-E_{I} / T}\right)\right],
\end{aligned}
$$

where $\Omega_{0}$ is a constant added to make the pressure of the vacuum vanishing (the bag constant can also be included on the right hand side if necessary). Here, while including electrons, we neglected the contribution of neutrinos. This properly describes the situation inside compact stars after the deleptonization had occurred, i.e., after the temperature of matter dropped so low 
that the neutrino mean free path became larger than a typical stellar size. In protoneutron stars this happens in less than a minute after their birth.

The sum in the second line of Eq. (76) runs over all (6 quark and 6 antiquark) quasiparticles. The dispersion relations and the degeneracy factors of the quasiparticles read

$$
\begin{aligned}
E_{u b}^{ \pm} & =E(p) \pm \mu_{u b}, & & {[\times 1] } \\
E_{d b}^{ \pm} & =E(p) \pm \mu_{d b}, & & {[\times 1] } \\
E_{\Delta^{ \pm}}^{ \pm} & =E_{\Delta}^{ \pm}(p) \pm \delta \mu . & & {[\times 2] }
\end{aligned}
$$

These can be obtained in a rather straightforward way from the quark propagator. Here, the following shorthand notation was introduced:

$$
\begin{aligned}
E(p) & \equiv \sqrt{\mathbf{p}^{2}+m^{2}} \\
E_{\Delta}^{ \pm}(p) & \equiv \sqrt{[E(p) \pm \bar{\mu}]^{2}+\Delta^{2}} \\
\bar{\mu} & \equiv \frac{\mu_{u r}+\mu_{d g}}{2}=\frac{\mu_{u g}+\mu_{d r}}{2}=\mu-\frac{\mu_{e}}{6}+\frac{\mu_{8}}{3} \\
\delta \mu & \equiv \frac{\mu_{d g}-\mu_{u r}}{2}=\frac{\mu_{d r}-\mu_{u g}}{2}=\frac{\mu_{e}}{2}
\end{aligned}
$$

The thermodynamic potential that determines the pressure of quark matter, $\Omega_{\text {phys }}=-P$, is obtained from the effective potential $\Omega$ in Eq. (76) after substituting the values of $\mu_{8}, \mu_{e}, m$ and $\Delta$ which solve the color and electric charge neutrality conditions, i.e.,

$$
n_{8} \equiv-\frac{\partial \Omega}{\partial \mu_{8}}=0, \quad \text { and } \quad n_{Q} \equiv-\frac{\partial \Omega}{\partial \mu_{e}}=0,
$$

as well as the gap equations, i.e.,

$$
\frac{\partial \Omega}{\partial m}=0, \quad \text { and } \quad \frac{\partial \Omega}{\partial \Delta}=0
$$

Only a solution that satisfies Eqs. (84) and (85) can correspond to a neutral ground state of quark matter. If there exist several such solutions, it is the solution which gives the highest value of the pressure that gives the ground state. 


\subsubsection{Three regimes in neutral matter}

In studying neutral two-flavor quark matter, it was found that there exist three qualitatively different dynamical regimes which differ by the strength of diquark coupling, as well as by the properties of the ground state 69, 73.

The first regime corresponds to weak diquark coupling. In this case, a weak Cooper pairing is completely suppressed by the mismatch between the Fermi momenta of the up and down quarks. In other words, the appearance of the 2SC phase is in conflict with the constraint of charge neutrality. The ground state of neutral matter corresponds to the normal phase in this regime. One should note, though, that a much weaker spin-1 pairing between quarks of same flavor is not forbidden in such neutral matter. In fact, if the temperature is sufficiently low, two independent spin-1 condensates, one made of up quarks and the other made of down quarks, are inevitable.

The other limiting case is the strongly coupled regime. It is clear that, if the value of the diquark coupling is sufficiently large, the color condensation could be made as strong as needed to overcome a finite mismatch between the Fermi surfaces of pairing quarks. In this argument, it is taken into account that the charge neutrality constraint is only slightly affected by the value of the diquark coupling. This is the case in all models that have been studied so far. In this regime, the ground state is in the 2SC phase, and the neutrality has little effect. Here, it might be appropriate to mention that the unpaired blue up and blue down quarks of the 2SC phase cannot produce any additional spin-1 condensate. The main reason is that their chemical potentials are badly mismatched.

In Fig. 4 the dispersion relations of quasiparticles in the $2 \mathrm{SC}$ phase (i.e., in the strongly coupled regime) and in the normal quark matter phase (i.e., in the weakly coupled regime) are shown by the solid and dashed lines, respectively. The dispersion relations of unpaired blue quasiparticles are not shown. It should be clear, however, that they are similar to those in the normal phase, albeit slightly shifted in the horizontal direction.

We see from Fig. 4 that there are two types of gapped quasiparticles in the spectrum of the $2 \mathrm{SC}$ phase. In fact, each of them is a doublet with respect to the unbroken $\mathrm{SU}(2)_{c}$ gauge group. One of the doublets has the gap $\Delta+\delta \mu$, and the other has the gap $\Delta-\delta \mu$, where $\delta \mu \equiv \mu_{e} / 2$ and $\Delta$ are the solutions of Eqs. (84) and (85) that describe the ground state. When

the mismatch parameter $\delta \mu$ is vanishing, two types of quasiparticles become degenerate. This corresponds to the ideal 2SC phase, considered in the first 

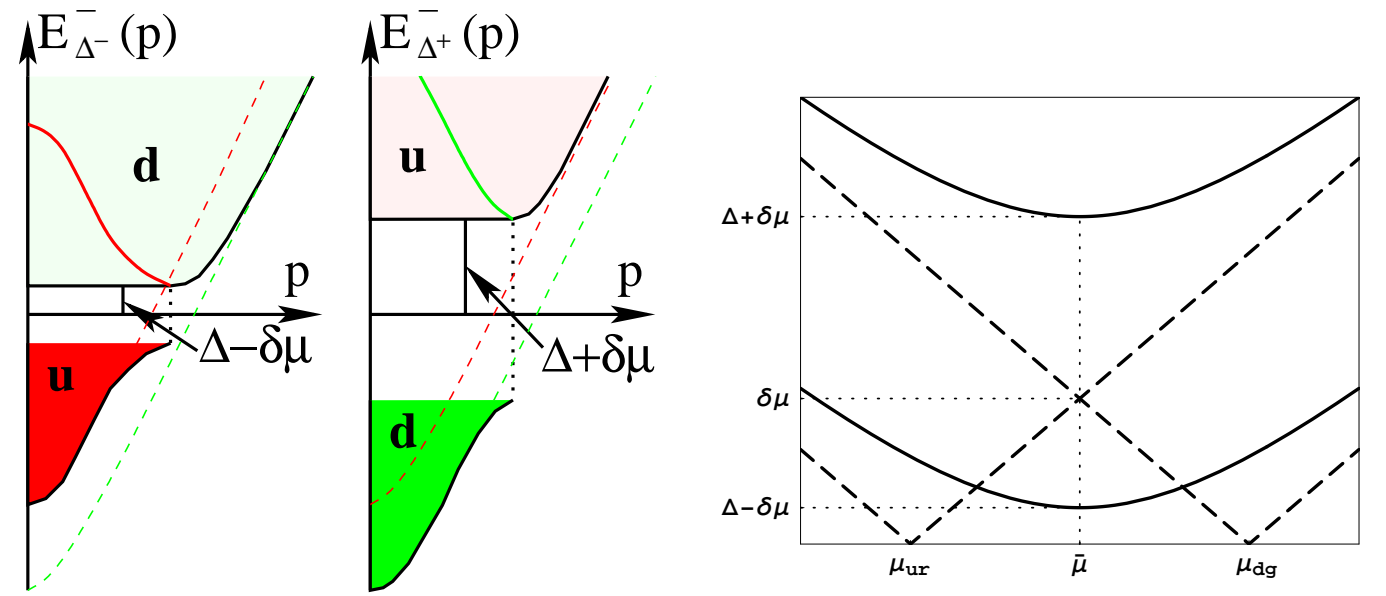

Figure 4: The dispersion relations of quark quasiparticles in the 2SC phase (solid lines) and the normal quark matter phase (dashed lines). The lowenergy part of the dispersion relations is shown in the right panel.

lecture.

The actual values of the coupling constants, which define the strongly and weakly coupled dynamical regimes, can differ very much from one effective model of QCD to another. Thus, it is convenient to use the value of the following dimensionless ratio $\Delta / \delta \mu$ instead of the value of the diquark coupling constant in a quantitative definition of such regimes. In this ratio, only the value of the gap $\Delta$ is strongly sensitive to the coupling constant.

From our classification above, it should be clear that $\Delta / \delta \mu=0$ corresponds to the weakly coupled regime. This should not be confused with the $G_{D}=0$ case. In fact, in any model, there should exist a critical value $G_{D}^{(1)}$ such that $\Delta / \delta \mu=0$ when $G_{D}<G_{D}^{(1)}$. As we shall see below, the strongly coupled regime is given by $\Delta / \delta \mu>1$. This makes sense after noting that the values of the two gaps in the 2SC phase, $\Delta+\delta \mu$ and $\Delta-\delta \mu$, are positive when $\Delta / \delta \mu>1$ (see Fig. (4)).

It turns out, that there also exists an intermediate regime, in which the diquark coupling is neither too weak nor too strong. In this third regime, the ground state is given by the so-called gapless $2 \mathrm{SC}$ phase [69, 73]. This phase corresponds to the ratio $\Delta / \delta \mu$ in the range from 0 to 1 (i.e., $0<\Delta / \delta \mu<1$ ). This regime will be discussed in more detail in the next subsection. 


\subsubsection{Gapless 2SC phase}

In the case of the NJL model used in Refs. 69, 73, the intermediate dynamical regime with $0<\Delta / \delta \mu<1$ is realized when the strength of the diquark coupling is given by $0.7 \lesssim \eta \lesssim 0.8$ where $\eta \equiv G_{D} / G_{S}$. To be specific, below we use $\eta=0.75$.

Let us start from a brief discussion of the neutrality conditions in Eq. (84). At zero temperature, they are satisfied approximately when $\mu_{8} \approx 0$ and

$$
\begin{aligned}
\mu_{e} & \approx \frac{3}{5} \mu, \text { for } \Delta>\delta \mu, \\
\Delta & \approx \sqrt{\frac{\mu_{e}^{2}}{4}-\left(\frac{\left(\mu-2 \mu_{e} / 3\right)^{3}-\mu_{e}^{3}}{6\left(\mu-\mu_{e} / 6\right)^{2}}\right)^{2}}, \quad \text { for } \quad \Delta \leq \delta \mu .
\end{aligned}
$$

It should be noted that the solutions in the two regions of parameters, $\Delta>$ $\delta \mu$ and $\Delta \leq \delta \mu$, are very different. One could check that this reflects a qualitatively different nature of the quasiparticle spectra in the two regions. While all the modes described by the dispersion relations in Eq. (79) are gapped when $\Delta>\delta \mu$, there appear a gapless doublet mode when $\Delta \leq \delta \mu$.

After imposing neutrality, the chemical potentials $\mu_{e}$ and $\mu_{8}$ are not free parameters, but (implicit) functions of $\Delta$. By making use of these functions $\mu_{e}=\mu_{e}(\Delta)$ and $\mu_{8}=\mu_{8}(\Delta)$ in Eq. (76)), one can define the effective potential for neutral matter as a function of the gap parameter $\Delta$. This potential is

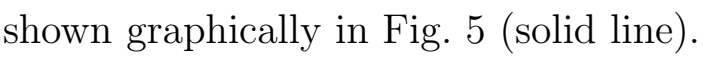

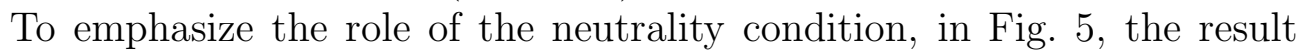
for the effective potential of non-neutral quark matter at a fixed value of the electron chemical potential $\mu_{e} \approx 148 \mathrm{MeV}$ is also shown (dashed line). The chemical potential $\mu_{e}$ is chosen so that the electric charge density vanishes when the value of the gap corresponds to the maximum of the potential. This effective potential (labelled " $\mu_{e}=148 \mathrm{MeV}$ ") describes negatively charged matter to the left from the maximum, and positively charged matter to the right from the maximum. On both sides, it is unphysical because of a large (infinite in an infinite volume) contribution of the Coulomb energy, see Eq. (64), that was neglected in Eq. (766). It is fair to note that the charged minima of the dashed line may become physically important if mixed phases are allowed [80, 39, 86.

From the location of the minimum of the effective potential for neutral

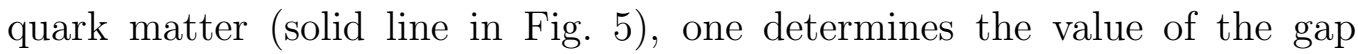
parameter in the ground state, $\Delta \approx 68 \mathrm{MeV}$. It appears that $\Delta<\delta \mu \equiv$ 


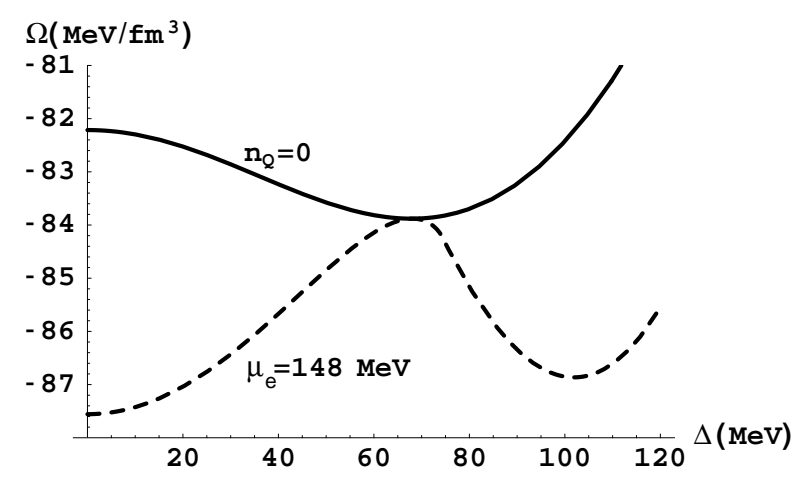

Figure 5: The effective potential as a function of the diquark gap $\Delta$ calculated at a fixed value of the electron chemical potential $\mu_{e} \approx 148 \mathrm{MeV}$ (dashed line), and the effective potential defined along the neutrality line (solid line). The results are plotted for $\mu=400 \mathrm{MeV}$ and $G_{D}=\eta G_{S}$ with $\eta=0.75$.

$\mu_{e} / 2 \approx 74 \mathrm{MeV}$ in such a ground state, i.e., it corresponds to the intermediate regime in our classification. The low energy quasiparticle spectrum in this phase contains additional (as compared to the 2SC phase) gapless modes 69, 73. This is also seen clearly from the analytical expressions in Eqs. (77)(179). Graphically, the dispersion relations are shown in Fig. 6. These should be compared with the corresponding quasiparticle dispersion relations in the 2SC phase in Fig. 4 .

The most remarkable property of the quasiparticle spectrum in the g2SC phase is that the low energy excitations $(E \ll \delta \mu-\Delta)$ are very similar to those in the normal phase. The only difference is that the values of the chemical potentials of the free up and down quarks are replaced by $\mu^{ \pm} \equiv$ $\bar{\mu} \pm \sqrt{(\delta \mu)^{2}-\Delta^{2}}$. This simple observation may suggest that the low energy (large distance scale) properties of the g2SC phase are similar to those in the normal phase [7]. For example, the Debye screening mass of the gluons of the $\mathrm{SU}(2)_{c}$ subgroup should be nonzero. The latter, in fact, should be proportional to the density of the gapless modes 90],

$$
m_{1, D}^{2} \simeq \frac{2 \alpha_{s}}{\pi}\left(\frac{\left(\mu^{-}\right)^{2} \delta \mu}{\sqrt{(\delta \mu)^{2}-\Delta^{2}}}+\frac{\left(\mu^{+}\right)^{2} \delta \mu}{\sqrt{(\delta \mu)^{2}-\Delta^{2}}}\right) \theta(\delta \mu-\Delta)
$$

Note that the corresponding value for the Debye screening mass in the or- 


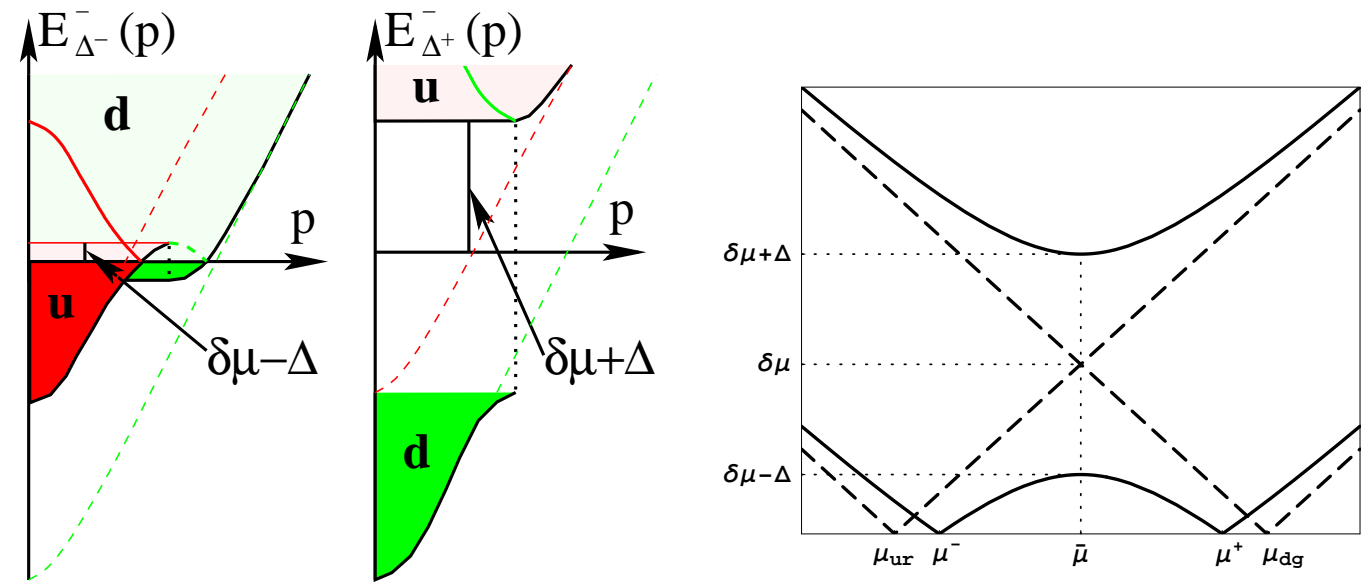

Figure 6: The dispersion relations of quark quasiparticles in the g2SC phase (solid lines) and the normal quark matter phase (dashed lines). The lowenergy part of the dispersion relations is shown in the right panel.

dinary 2SC phase is vanishing 91]. The overall coefficient in Eq. (88) can be fixed by matching the value of the Debye screening mass with the known result in the normal phase. The Meissner screening mass of the gluons of the unbroken $\mathrm{SU}(2)_{c}$ subgroup is vanishing, $m_{1, M}^{2}=0$, as it should be [90].

The magnetic properties of the g2SC phase are nothing like those in the normal phase. The values of the Meissner screening masses of five gluons are imaginary, indicating a chromomagnetic instability in dense two-flavor quark matter [90]. This instability may lead to a gluon condensation, and possibly to breaking the rotational or the translational symmetry in the true ground state of quark matter. This could be a completely new state, or this could be one of the states proposed earlier. For example, it may be a state with deformed quark Fermi surfaces [92, or it may turn into the LarkinOvchinnikov-Fulde-Ferrell state 93, which was discussed in Ref. 94] in the context of quark matter. To make a certain statement, further studies in this direction are needed.

\subsubsection{Finite temperature properties}

The finite temperature properties of neutral quark matter in the intermediate coupling regime are also very unusual [73, 74]. The most striking are the results for the temperature dependence of the gap parameter for various 
values of the diquark coupling strengths. This is demonstrated by the plot in Fig. (7. As one can see, the results for not very strong coupling are very unusual. The gap function may take a finite value at nonzero temperatures even though it is exactly zero at zero temperature. This possibility comes about only because of the neutrality condition which introduces a mismatch between the Fermi momenta of pairing quarks. The thermal excitations smear the Fermi surfaces of the up and down quarks, and this opens the possibility of Cooper pairing which is forbidden at zero temperature. With increasing the temperature, the thermal fluctuations will eventually become too strong, and the pairing will be destroyed.
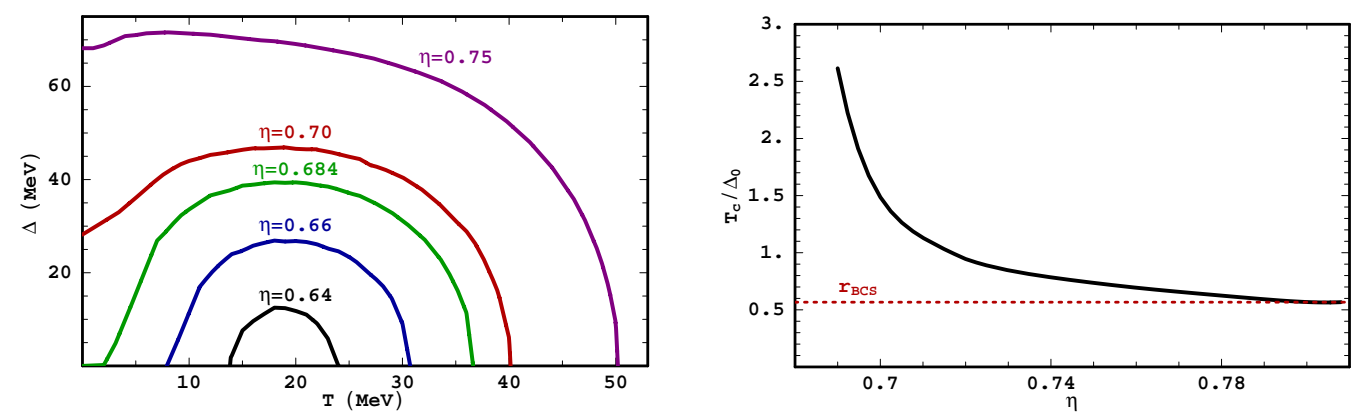

Figure 7: The temperature dependence of the diquark gap in neutral quark matter calculated for several values of the diquark coupling strength $\eta=G_{D} / G_{S}$ (left panel), and the ratio of the critical temperature to the zero temperature gap in neutral quark matter as a function of the coupling strength $\eta$ (right panel).

By looking at the results in the left panel of Fig. [7] one should immediately realize that the value of the ratio of the critical temperature $T_{c}$ to the value of the gap at zero temperature $\Delta_{0}$ is not a universal number. In contrast to the Bardeen-Copper-Schrieffer theory, the ratio $T_{c} / \Delta_{0}$ in the neutral phase depends on the diquark coupling constant. This is shown explicitly in the right panel in Fig. 17. As we see, the ratio $T_{c} / \Delta_{0}$ can be arbitrarily large, and even remain strictly infinite for a range of coupling strengths.

\subsection{Gapless color-flavor locked phase}

While considering a realistic model of strange quark matter, one should take into account that the strange quark mass is much larger than the masses 
of the light up and down quarks. Most likely, the actual value of strange quark mass in dense medium should be in the range between about $100 \mathrm{MeV}$ and $500 \mathrm{MeV}$. This is not negligible in comparison with a typical value of the quark chemical potential $\mu \simeq 500 \mathrm{MeV}$ at densities existing in compact stars.

In application to the Cooper pairing dynamics responsible for color superconductivity, the main effect of a non-vanishing strange quark mass is a reduction of the strange quark Fermi momentum,

$$
k_{F}^{(s)}=\sqrt{\mu^{2}-m_{s}^{2}} \simeq \mu-\frac{m_{s}^{2}}{2 \mu} .
$$

The magnitude of the reduction is approximately given by the value of $m_{s}^{2} / 2 \mu$. This quantity plays the role of a mismatch parameter in three-flavor quark matter, which is similar to $\delta \mu \equiv \mu_{e} / 2$ in two-flavor quark matter. For obvious reasons, this mismatch should interfere with Cooper pairing between strange and non-strange quarks.

Because of a nonzero strange quark mass, the diquark condensate of the ideal CFL phase (47) may get distorted as follows [68, 75]:

$$
\left\langle\psi_{L, i}^{a, \alpha} \epsilon_{\alpha \beta} \psi_{L, j}^{b, \beta}\right\rangle=-\left\langle\psi_{R, i}^{a, \dot{\alpha}} \epsilon_{\dot{\alpha} \dot{\beta}} \psi_{R, j}^{b, \dot{\beta}}\right\rangle \sim \Delta_{1} \varepsilon_{i j 1} \epsilon^{a b 1}+\Delta_{2} \varepsilon_{i j 2} \epsilon^{a b 2}+\Delta_{3} \varepsilon_{i j 3} \epsilon^{a b 3}+\cdots,
$$

where the ellipsis denote terms symmetric in color and flavor. As in the ideal CFL phase, they are small and not crucial for the qualitative understanding of strange quark matter [78].

The three gap parameters $\Delta_{1}, \Delta_{2}$ and $\Delta_{3}$ in Eq. (90) describe downstrange, up-strange and up-down condensates of Cooper pairs, respectively. A nonzero value of $m_{s}^{2} / 2 \mu$ affects first and foremost the pairing between the strange and non-strange quarks, i.e., the gap parameters $\Delta_{1}$ and $\Delta_{2}$. Because of the color-flavor locking, preserved in the diquark condensate (90), this translates into a special role played by the blue quarks.

By starting from the massless case and gradually increasing the value of strange quark mass, one finds that the CFL phase stays robust until a critical value of the control parameter $m_{s}^{2} / 2 \mu \simeq \Delta$ is reached [75]. Here, $\Delta$ is the gap parameter in the CFL phase. (Strictly speaking, $\Delta \equiv \Delta_{1}=\Delta_{2} \approx \Delta_{3}$ in the CFL phase when $m_{s} \neq 0$.) Above the critical value, the charge neutrality cannot be accommodated in the CFL phase, and a new gapless phase appears 75. 
A nice feature of the CFL phase is that it stays almost automatically electrically neutral [95. The reason is that Cooper pairing in the CFL phase helps to enforce the equal number densities of all three quark flavors, $n_{u}=$ $n_{d}=n_{s}$. Since the sum of the charges of the up, down and strange quarks add up to zero, this insures that the electric charge density is vanishing, $n_{Q}=\frac{2}{3} n_{u}-\frac{1}{3} n_{d}-\frac{1}{3} n_{s}=0$. This is exactly what happens in the CFL phase even at nonzero, but sub-critical values of the strange quark mass.

It is the color rather than the electric charge neutrality that plays an important role in three-flavor quark matter at a nonzero value of $m_{s}^{2} / 2 \mu$. It has been mentioned above that, because of the color-flavor locking, the blue quarks have a special status in the Cooper pairing dynamics. In order to avoid the violation of the color neutrality, then, a nonzero compensating value of the color chemical potential $\mu_{8} \simeq-m_{s}^{2} / 2 \mu$ is needed [67]. (Note that, in QCD with dynamical gluons, a nonzero value of the gluon condensate $\left\langle A_{0}^{8}\right\rangle$ will be generated instead 88 .)

It was shown in Ref. [75] that the CFL phase becomes gapless when $m_{s}^{2} / 2 \mu>\Delta$. In essence, the mechanism is the same as in two-flavor quark matter, discussed earlier in this lecture. In order to get a slightly better understanding, it is instructive to consider how the strange quark mass interferes with the pairing between blue down quarks and green strange quarks [75]. After taking into account the shift of the strange quark Fermi momentum in Eq. (89), the effective values of the chemical potentials of blue down and green strange quarks become

$$
\begin{aligned}
& \mu_{d b} \simeq \mu-\frac{2}{3} \mu_{8} \simeq \mu+\frac{m_{s}^{2}}{3 \mu} \\
& \mu_{s g}^{\mathrm{eff}} \simeq \mu+\frac{1}{3} \mu_{8}-\frac{m_{s}^{2}}{2 \mu} \simeq \mu-\frac{2 m_{s}^{2}}{3 \mu},
\end{aligned}
$$

where we took into account that $\mu_{8} \simeq-m_{s}^{2} / 2 \mu$. Up to an effective shift of the strange quark chemical potential, these expressions for the quark chemical potentials are similar to those in Eqs. (67)-(70) where $\mu_{e}=0$. In analogy with the two-flavor case, see Eq. (883), we define

$$
\delta \mu=\frac{\mu_{d b}-\mu_{s g}^{\mathrm{eff}}}{2}=\frac{m_{s}^{2}}{2 \mu},
$$

as a formal value of the mismatch parameter. As soon as $\delta \mu>\Delta$, one should get the gapless modes in the quasiparticle spectrum. Their dispersion relations are given by the same expression (799), except that $\bar{\mu} \rightarrow\left(\mu_{d b}+\mu_{s g}^{\text {eff }}\right) / 2$. 
a)

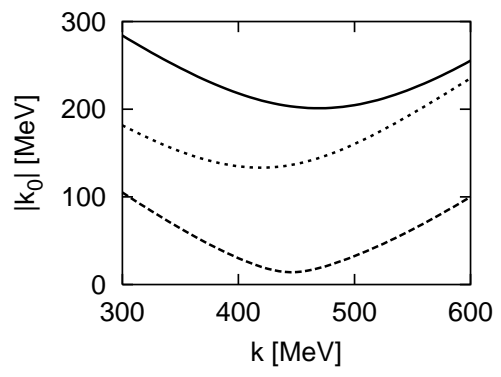

C)

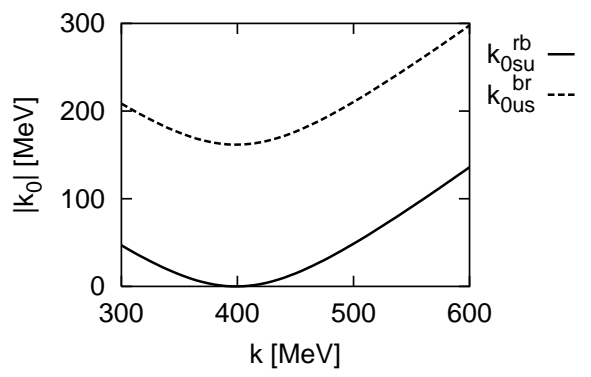

b)

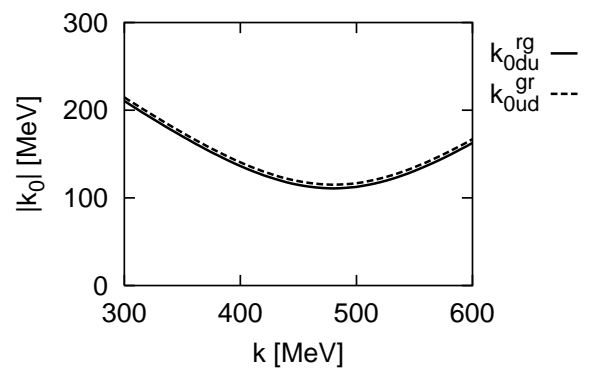

d)

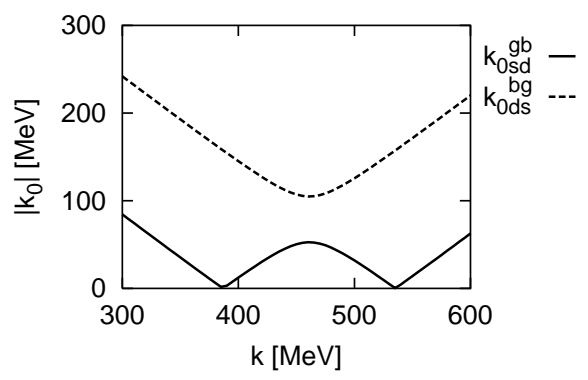

Figure 8: The quasiparticle dispersion relations for electric and color neutral color superconducting quark matter at $T=0, \mu=500 \mathrm{MeV}$, and $m_{s}=$ $400 \mathrm{MeV}$, see Ref. 78.

A typical result for the low-energy dispersion relations of all nine quasiparticle are shown in Fig. 8 which is taken from Ref. [78. It should be noted that the two dispersion relations in panel (d) are qualitatively the same as those in Fig. 6 in the gapless 2SC case.

Physical properties of the gCFL phase are very different from those of the CFL phase. The presence of gapless quasiparticle modes with a density of states proportional to $\mu^{2}$ has a large effect on the thermodynamics as well as on the transport. In contrast to the CFL phase which is an insulator, the gCFL phase is a metal with a nonzero number density of electrons. Also, the neutrino emissivity rate from the gCFL phase should be rather high. It is dominated by the $\beta$-processes: $\tilde{d}_{b} \rightarrow \tilde{u}_{b}+e^{-}+\bar{\nu}_{e}$ and $\tilde{u}_{b}+e^{-} \rightarrow \tilde{d}_{b}+\nu_{e}$, where $\tilde{u}_{b}$ and $\tilde{d}_{b}$ denote the gapless quasiparticles whose dispersion relations are represented by the solid lines in panels (c) and (d) in Fig. 8. Relatively large contributions to the neutrino emissivity may also come from the processes with additional gluons: $\tilde{s}_{r, g} \rightarrow \tilde{u}_{b}+e^{-}+\bar{\nu}_{e}+g_{4,5,6,7}$ and $\tilde{u}_{b}+e^{-} \rightarrow \tilde{s}_{r, g}+\nu_{e}+$ $g_{4,5,6,7}$, where $g_{4,5,6,7}$ denotes one of the gluons described by the gauge fields 
$A_{\mu}^{4}, A_{\mu}^{5}, A_{\mu}^{6}$ or $A_{\mu}^{7}$

\subsection{Mixed phases with color superconducting compo- nents}

In addition to homogeneous (one-component) phases, one could also study various mixed phases of dense quark matter. The neutrality in such phases is imposed not locally, but globally (i.e., on average). For example, one can

construct a phase which is made of alternating layers of different coexisting components. While each of the component may have a nonzero charge density, the average charge density of the whole construction is vanishing. The Coulomb energy in a mixed phase is nonzero, but it does not grow with the volume of the system. There is also a contribution to the total energy that comes from surfaces separating components of the mixed phase [80, 39, 86. If the corresponding surface tension is sufficiently large, the mixed phase is disfavored energetically, and therefore it cannot appear. If, on the other hand, the surface tension is small, the mixed phase can be the ground state of matter, replacing a homogeneous gapless phase.

\subsubsection{Gibbs construction}

Here is a brief introduction to the general method of constructing mixed phases by imposing the Gibbs conditions of equilibrium [96, 97. From the physical point of view, the Gibbs conditions enforce the mechanical as well as chemical equilibrium between different components in a mixed phase. This is achieved by requiring that the pressure of different components inside the mixed phase are equal, and that the chemical potentials $\left(\mu\right.$ and $\left.\mu_{e}\right)$ are the same across the whole mixed phase. For example, in application to the mixed phase made of the normal and the 2SC quark components [39], these conditions read

$$
\begin{aligned}
P^{(N Q)}\left(\mu, \mu_{e}\right) & =P^{(2 S C)}\left(\mu, \mu_{e}\right) \\
\mu & =\mu^{(N Q)}=\mu^{(2 S C)} \\
\mu_{e} & =\mu_{e}^{(N Q)}=\mu_{e}^{(2 S C)}
\end{aligned}
$$

It is easy to visualize these conditions by plotting the pressure surfaces as functions of chemical potentials $\left(\mu\right.$ and $\left.\mu_{e}\right)$ for the components of the mixed phase. Graphically, this is shown in Fig. 9. The Gibbs conditions are 
automatically satisfied along the intersection line of the pressure surfaces (dark solid line in Fig. 9).

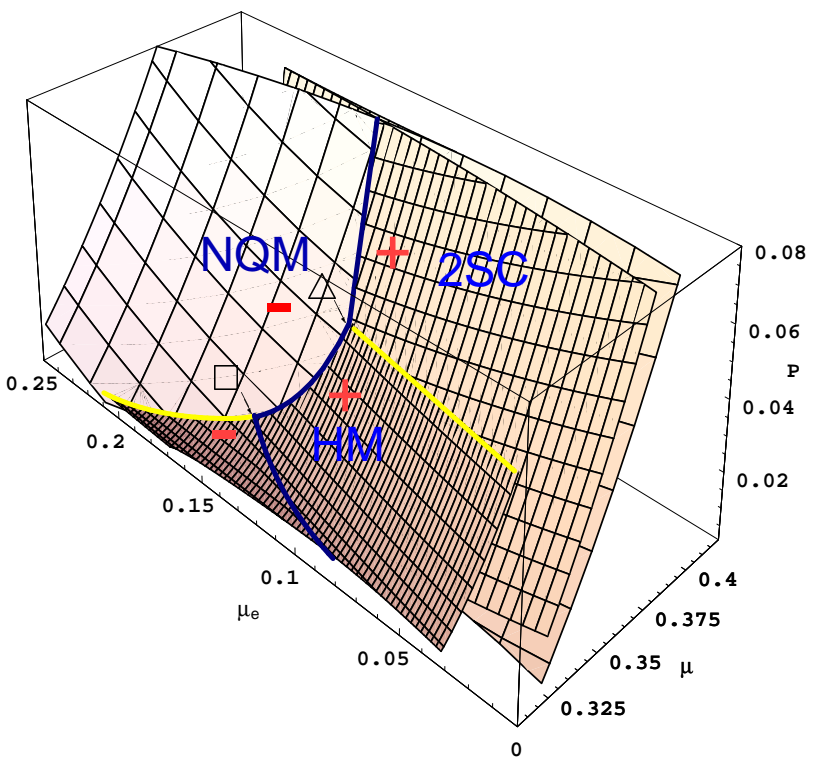

Figure 9: Pressure as a function of $\mu \equiv \mu_{B} / 3$ and $\mu_{e}$ for the hadronic phase (at the bottom), for the 2SC phase (on the right in front) and the normal quark phase (on the left). The dark thick line follows the neutrality line in the hadronic phase, and in two mixed phases: (i) the mixed phase of hadronic and normal quark matter; and (ii) the mixed phase of normal and color superconducting quark matter.

Different components of the mixed phase occupy different volume fractions in space. To describe this quantitatively, it is convenient to introduce the volume fraction of each component. As an example, let us consider the quark mixed phase made of the normal and the 2SC component 39. We denote the volume fraction of the normal phase by $\chi_{2 S C}^{N Q} \equiv V_{N Q} / V$ (in general, the notation $\chi_{B}^{A}$ means "the volume fraction of phase $\mathrm{A}$ in a mixture with phase B"). Then, the volume fraction of the 2SC phase is given by $\chi_{N Q}^{2 S C}=\left(1-\chi_{2 S C}^{N Q}\right)$. From the definition, it is clear that $0 \leq \chi_{B}^{A} \leq 1$.

The average electric charge density of the mixed phase is determined by the charge densities of its components taken in the proportion of the corresponding volume fractions. Thus,

$$
n_{e}^{(M P)}\left(\mu, \mu_{e}\right)=\chi_{2 S C}^{N Q} n_{e}^{(N Q)}\left(\mu, \mu_{e}\right)+\left(1-\chi_{2 S C}^{N Q}\right) n_{e}^{(2 S C)}\left(\mu, \mu_{e}\right) .
$$


If the charge densities of the two components have opposite signs, one can impose the global charge neutrality condition, $n_{e}^{(M P)}=0$. Otherwise, a neutral mixed phase could not exist. In the case of quark matter, the charge density of the normal quark phase is negative, while the charge density of the 2SC phase is positive along the line of the Gibbs construction (dark solid line in Fig. 91). Therefore, a neutral mixed phase exists. The volume fractions of its components are

$$
\begin{aligned}
\chi_{2 S C}^{N Q} & =\frac{n_{e}^{(2 S C)}}{n_{e}^{(2 S C)}-n_{e}^{(N Q)}}, \\
\chi_{N Q}^{2 S C} & \equiv 1-\chi_{2 S C}^{N Q}=\frac{n_{e}^{(N Q)}}{n_{e}^{(N Q)}-n_{e}^{(2 S C)}} .
\end{aligned}
$$

After the volume fractions have been determined from the condition of the global charge neutrality, the energy density of the corresponding mixed phase can also be calculated,

$$
\varepsilon^{(M P)}\left(\mu, \mu_{e}\right)=\chi_{2 S C}^{N Q} \varepsilon^{(N Q)}\left(\mu, \mu_{e}\right)+\left(1-\chi_{2 S C}^{N Q}\right) \varepsilon^{(2 S C)}\left(\mu, \mu_{e}\right) .
$$

This is essentially all what one needs in order to construct the equation of state of the mixed phase.

\subsubsection{Surface tension and Coulomb forces}

In the above construction, the effects of the Coulomb forces and the surface tension between different components of the mixed phase were neglected. In reality, these might be important. In particular, the balance between the Coulomb forces and the surface tension determines the geometries of different components inside the mixed phase.

In the mixed phase made of the normal and 2SC components, for example, nearly equal volume fractions of the two quark phases are likely to form alternating layers (slabs) of matter. The energy cost per unit volume to produce such layers scales as $\sigma^{2 / 3}\left(n_{e}^{(2 S C)}-n_{e}^{(N Q)}\right)^{2 / 3}$ where $\sigma$ is the surface tension 98. Therefore, the quark mixed phase is a favorable phase of matter only if the surface tension is not too large. The estimates of Ref. 80 show that the value of $\sigma$ at the boundary between the normal and the 2SC phases is only about 5 to $10 \mathrm{MeV} / \mathrm{fm}^{2}$. The maximum value of $\sigma$ allowed in the mixed phase, on the other hand, is of order 10 to $15 \mathrm{MeV} / \mathrm{fm}^{2}$ when the value of the quark chemical potential is in the range between 400 and $500 \mathrm{MeV}$. 
Therefore, one should conclude that the mixed phase is more favorable than the gapless 2SC phase.

Here, it is fair to mention that the conclusion of Ref. 80 may not be final yet. The reason is that the thickness of the layers, which scales as

$\sigma^{1 / 3}\left(n_{e}^{(2 S C)}-n_{e}^{(N Q)}\right)^{-2 / 3}[98$, is estimated to be of order 5 to $10 \mathrm{fm}$ in the mixed phase [80, 39]. This is comparable to the value of the Debye screening length in each of the two quark phases. Therefore, the effects of charge screening, which have been neglected so far and which are known to increase the surface energy [99, may still change the conclusion.

\subsection{Summary}

In recent years there was a lot of progress in understanding the mechanism of color superconductivity and in studying physical properties of various possible color superconducting phases of dense quark matter. At present, one can say that the QCD theory of strong interactions at asymptotic densities is most likely to have a color superconducting ground state. Unfortunately, however, one cannot say with certainty what is the critical value of the baryon density at which color superconductivity first appears. Because of this, one does not know for sure if color superconducting phases could appear at highest densities existing in the Universe.

In nature, very dense baryonic matter exists in compact stars. Thus, it is possible that color superconductivity exists in the central regions of the heaviest compact stars. This conjecture has not yet been (dis-)proved, and it should come under scrutiny in the near future. In this connection, it might be appropriate to mention that there were recent observations on the cooling [100] of one neutron star and the radius [101] of another that led the authors to suggest the presence of quark matter. These suggestions have been disputed 102, 103. However, the current situation, where there is no unambiguous evidence that deconfined quarks play a role in compact stars, may well change as further observations are made.

One of the main consequences of color superconductivity in dense matter is the appearance of an energy gap in the quasiparticle spectrum. In QCD, the typical value of the gap is $100 \mathrm{MeV}$. The presence of such a large gap is likely to affect many physical properties of matter, which in their turn should be reflected in the observational properties of stars. It is of great interest, therefore, to perform a systematic study of the properties of dense baryonic matter under conditions typical for the interior of stars. The goal 
of such studies should be a clear theoretical prediction of the most favorable phases of matter inside stars. Also, one should work out the key physical properties of different phases that could have an unambiguous imprint on the observational data from stars. Such a theoretical basis would be crucial in an attempt to discover new (e.g., quark) states of matter in nature.

The current understanding of color superconducting phases of QCD at large baryon chemical potential $\mu$ may also turn out to be useful for developing alternative methods to treat QCD at nonzero $\mu$ on the lattice [104, 105. As is known, there are not so many methods to study QCD from first principles. One of them is the method of lattice calculations [106. As of now, the lattice calculations have produced many results for QCD at finite temperatures and vanishing baryon chemical potential $\mu=0$ [107. There are also some results at nonzero but small values of the baryon chemical potential 108. The lattice calculations at large nonzero $\mu$ are problematic because of the famous sign problem. At $\mu \neq 0$, the fermion determinant in the functional integral becomes complex, and the standard numerical techniques, which rely on a probability interpretation of the integrand, cannot be easily applied. Thus, new approaches and new ideas are needed.

Building on the knowledge gained from lattice calculations at nonzero temperature as well as from the effective models of QCD at nonzero chemical potential, one would like to get eventually a complete QCD phase diagram in the plane of temperature and quark chemical potential. The knowledge

of such a diagram is not only of a theoretical importance. Different regions of the phase diagram describe physical conditions that can be realized, for example, during the evolution of the early Universe or in the heavy ion collisions. There is also a region at nonzero baryon chemical potentials which is responsible for the physics of stars. In order to understand the corresponding physics, one first has to know the locations of the critical lines and the types of the phase transitions. Some basic features of the QCD diagram have already started to emerge [78, 79, 109, 110]. It will probably take many years before the complete phase diagram is understood in detail.

\section{Acknowledgements}

I would like to thank the organizers of the IARD 2004 conference and the organizers of the Helmholtz International Summer School and Workshop on Hot points in Astrophysics and Cosmology for their invitation to present these lectures. Also, I thank Michael Buballa, Valery Gusynin, Paul Ellis, 
Matthias Hanauske, Deog-Ki Hong, Mei Huang, Volodya Miransky, Micaela Oertel, Dirk Rischke, Stefan Rüster, Gordon Semenoff, and Rohana Wijewardhana for very fruitful collaboration on various topics in color superconductivity. This work was supported by Gesellschaft für Schwerionenforschung (GSI) and by Bundesministerium für Bildung und Forschung (BMBF).

\section{References}

[1] K. Hagiwara et al. [Particle Data Group Collaboration], Phys. Rev. D 66, 010001 (2002).

[2] D. Ivanenko and D.F. Kurdgelaidze, Astrofiz. 1, 479 (1965); Lett. Nuovo Cim. 2, 13 (1969); N. Itoh, Prog. Theor. Phys. 44, 291 (1970); F. Iachello, W.D. Langer, and A. Lande, Nucl. Phys. A 219, 612 (1974).

[3] H.D. Politzer, Phys. Rev. Lett. 30, 1346 (1973); D.J. Gross and F. Wilczek, Phys. Rev. D 8, 3633 (1973); Phys. Rev. D 9, 980 (1974).

[4] J.C. Collins and M.J. Perry, Phys. Rev. Lett. 34, 1353 (1975).

[5] J. I. Kapusta, Finite-temperature field theory, (University Press, Cambridge, 1989).

[6] A. Chodos, R. L. Jaffe, K. Johnson, C. B. Thorn, and V. F. Weisskopf, Phys. Rev. D 9, 3471 (1974); A. Chodos, R. L. Jaffe, K. Johnson, and C. B. Thorn, Phys. Rev. D 10, 2599 (1974).

[7] B. A. Freedman and L. D. McLerran, Phys. Rev. D 16, 1169 (1977).

[8] L.N. Cooper, Phys. Rev. 104, 1189 (1956).

[9] J. Bardeen, L.N. Cooper, and J.R. Schrieffer, Phys. Rev. 106, 162 (1957); Phys. Rev. 108, 1175 (1957).

[10] K. Rajagopal and F. Wilczek, hep-ph/0011333; D. K. Hong, Acta Phys. Polon. B 32, 1253 (2001); M. Alford, Ann. Rev. Nucl. Part. Sci. 51, 131 (2001); T. Schäfer, hep-ph/0304281; D. H. Rischke, Prog. Part. Nucl. Phys. 52, 197 (2004); H.-c. Ren, hep-ph/0404074, M. Huang, hep-ph/0409167. 
[11] B.C. Barrois, Nucl. Phys. B129, 390 (1977); S.C. Frautschi, in "Hadronic matter at extreme energy density", edited by N. Cabibbo and L. Sertorio (Plenum Press, 1980).

[12] D. Bailin and A. Love, Nucl. Phys. B190, 175 (1981); Nucl. Phys. B205, 119 (1982); Phys. Rep. 107, 325 (1984).

[13] M. Alford, K. Rajagopal, and F. Wilczek, Phys. Lett. B 422, 247 (1998); R. Rapp, T. Schäfer, E. V. Shuryak, and M. Velkovsky, Phys. Rev. Lett. 81, 53 (1998).

[14] Y. Nambu and G. Jona-Lasinio, Phys. Rev. 122, 345 (1961); Phys. Rev. 124, 246 (1961).

[15] G. W. Carter and D. Diakonov, Phys. Rev. D 60, 016004 (1999); J. Berges and K. Rajagopal, Nucl. Phys. B 538, 215 (1999); M. Buballa and M. Oertel, Nucl. Phys. A 703, 770 (2002); F. Gastineau, R. Nebauer, and J. Aichelin, Phys. Rev. C 65, 045204 (2002); M. Huang, P. F. Zhuang, and W. Q. Chao, Phys. Rev. D 65, 076012 (2002).

[16] M. Buballa, hep-ph/0402234.

[17] M. Huang, P. F. Zhuang, and W. Q. Chao, Phys. Rev. D 67, 065015 (2003).

[18] T. M. Schwarz, S. P. Klevansky, and G. Papp, Phys. Rev. C 60, 055205 (1999).

[19] D. J. Gross, R. D. Pisarski, and L. G. Yaffe, Rev. Mod. Phys. 53, 43 (1981); E. V. Shuryak, Nucl. Phys. B 203, 140 (1982); D. T. Son, M. A. Stephanov, and A. R. Zhitnitsky, Phys. Lett. B 510, 167 (2001).

[20] R. Casalbuoni, Z. Y. Duan, and F. Sannino, Phys. Rev. D 62, 094004 (2000).

[21] V. A. Miransky, I. A. Shovkovy, and L. C. R. Wijewardhana, Phys. Rev. D 62, 085025 (2000); Phys. Rev. D 64, 096002 (2001).

[22] D. H. Rischke, D. T. Son, and M. A. Stephanov, Phys. Rev. Lett. 87, 062001 (2001). 
[23] W. E. Brown, J. T. Liu, and H.-c. Ren, Phys. Rev. D 61, 114012 (2000); Q. Wang and D. H. Rischke, Phys. Rev. D 65, 054005 (2002).

[24] H. Vija and M.H. Thoma, Phys. Lett. B 342, 212 (1995).

[25] C. Manuel, Phys. Rev. D 53, 5866 (1996).

[26] V. A. Miransky, G. W. Semenoff, I. A. Shovkovy, and L. C. R. Wijewardhana, Phys. Rev. D 64, 025005 (2001); P. Jaikumar and I. Zahed, Phys. Rev. D 64, 014035 (2001).

[27] U. W. Heinz, Annals Phys. 168, 148 (1986).

[28] D. T. Son, Phys. Rev. D 59, 094019 (1999).

[29] D. K. Hong, V. A. Miransky, I. A. Shovkovy, and L. C. R. Wijewardhana, Phys. Rev. D 61, 056001 (2000); T. Schäfer and F. Wilczek, Phys. Rev. D 60, 114033 (1999); S. D. H. Hsu and M. Schwetz, Nucl. Phys. B572, $211(2000)$.

[30] R. D. Pisarski and D. H. Rischke, Phys. Rev. D 61, 051501 (2000); Phys. Rev. D 61, 074017 (2000).

[31] N. Iwamoto, Phys. Rev. Lett. 44, 1637 (1980).

[32] The pressure correction due to color superconductivity is determined, up to a sign, by the value of the effective potential at its minimum in V. A. Miransky, I. A. Shovkovy, and L. C. R. Wijewardhana, Phys. Lett. B 468, 270 (1999).

[33] I. A. Shovkovy and P. J. Ellis, Phys. Rev. C 66, 015802 (2002); C. Manuel, A. Dobado, and F. J. Llanes-Estrada, hep-ph/0406058

[34] G. Lugones and J. E. Horvath, Phys. Rev. D 66, 074017 (2002).

[35] M. Alford and S. Reddy, Phys. Rev. D 67 (2003) 074024.

[36] R. C. Tolman, Phys. Rev. 55, 364 (1939); J. R. Oppenheimer and G. M. Volkoff, Phys. Rev. 55, 374 (1939).

[37] S. Banik and D. Bandyopadhyay, Phys. Rev. D 67 (2003) 123003. 
[38] M. Baldo, M. Buballa, F. Burgio, F. Neumann, M. Oertel, and H.J. Schulze, Phys. Lett. B 562 (2003) 153.

[39] I. Shovkovy, M. Hanauske, and M. Huang, Phys. Rev. D 67, 103004 (2003).

[40] D. Blaschke, H. Grigorian, D.N. Aguilera, S. Yasui, and H. Toki, AIP Conf. Proc. 660 (2003) 209.

[41] M. Buballa, F. Neumann, M. Oertel, and I. Shovkovy, Phys. Lett. B 595, 36 (2004).

[42] A. R. Bodmer, Phys. Rev. D 4, 1601 (1971); E. Witten, Phys. Rev. D 30, 272 (1984); C. Alcock, E. Farhi, and A. Olinto, Astrophys. J. 310, 261 (1986).

[43] M. G. Alford, K. Rajagopal, and F. Wilczek, Nucl. Phys. B537, 443 (1999).

[44] I. A. Shovkovy and L. C. R. Wijewardhana, Phys. Lett. B 470, 189 (1999); T. Schäfer, Nucl. Phys. B575, 269 (2000).

[45] T. Schäfer, Nucl. Phys. A 728, 251 (2003).

[46] P. W. Anderson and N. Itoh Nature 256, 25 (1975).

[47] I. A. Shovkovy and P. J. Ellis, Phys. Rev. C 67, 048801 (2003).

[48] C. Vogt, R. Rapp, and R. Ouyed, Nucl. Phys. A 735, 543 (2004).

[49] J. Gasser and H. Leutwyler, Nucl. Phys. B 250, 465 (1985); Annals Phys. 158, 142 (1984); G. Ecker, Prog. Part. Nucl. Phys. 35, 1 (1995); A. Pich, Rept. Prog. Phys. 58, 563 (1995).

[50] D. K. Hong, M. Rho, and I. Zahed, Phys. Lett. B 468, 261 (1999).

[51] R. Casalbuoni and R. Gatto, Phys. Lett. B 464, 111 (1999).

[52] D. T. Son and M. A. Stephanov, Phys. Rev. D 61, 074012 (2000); Erratum: Phys. Rev. D 62, 059902 (2000). 
[53] K. Zarembo, Phys. Rev. D 62, 054003 (2000); S. R. Beane, P. F. Bedaque, and M. J. Savage, Phys. Lett. B 483, 131 (2000); V. A. Miransky, I. A. Shovkovy, and L. C. R. Wijewardhana, Phys. Rev. D 63, 056005 (2001).

[54] D. T. Son, M. A. Stephanov, and A. R. Zhitnitsky, Phys. Rev. Lett. 86, 3955 (2001).

[55] C. Manuel and M. H. G. Tytgat, Phys. Lett. B 479, 190 (2000); T. Schäfer, Phys. Rev. D 65, 074006 (2002).

[56] D. K. Hong, Phys. Lett. B 473, 118 (2000); Nucl. Phys. B 582, 451 (2000); G. Nardulli, Riv. Nuovo Cim. 25N3, 1 (2002); P. T. Reuter, Q. Wang, and D. H. Rischke, nucl-th/0405079.

[57] P. F. Bedaque and T. Schäfer, Nucl. Phys. A 697, 802 (2002).

[58] D. B. Kaplan and S. Reddy, Phys. Rev. D 65, 054042 (2002).

[59] A. Kryjevski, D. B. Kaplan, and T. Schäfer, hep-ph/0404290.

[60] V. A. Miransky and I. A. Shovkovy, Phys. Rev. Lett. 88, 111601 (2002); T. Schäfer, D. T. Son, M. A. Stephanov, D. Toublan, and J. J. M. Verbaarschot, Phys. Lett. B 522, 67 (2001).

[61] M. Iwasaki and T. Iwado, Phys. Lett. B 350, 163 (1995).

[62] T. Schäfer, Phys. Rev. D 62, 094007 (2000); M. G. Alford, J. A. Bowers, J. M. Cheyne, and G. A. Cowan, Phys. Rev. D 67, 054018 (2003); M. Buballa, J. Hošek, and M. Oertel, Phys. Rev. Lett. 90, 182002 (2003).

[63] A. Schmitt, Q. Wang, and D. H. Rischke, Phys. Rev. Lett. 91, 242301 (2003); Phys. Rev. D 69, 094017 (2004)

[64] A. Schmitt, nucl-th/0405076.

[65] D. Blaschke, D. N. Voskresensky, and H. Grigorian, astro-ph/0403171

[66] T. Schäfer and F. Wilczek, Phys. Rev. Lett. 82, 3956 (1999); Phys. Rev. D 60, 074014 (1999).

[67] M. Alford and K. Rajagopal, JHEP 0206, 031 (2002). 
[68] A.W. Steiner, S. Reddy, and M. Prakash, Phys. Rev. D 66, 094007 (2002).

[69] I. Shovkovy and M. Huang, Phys. Lett. B 564, 205 (2003).

[70] E. Gubankova, W.V. Liu, and F. Wilczek, Phys. Rev. Lett. 91, 032001 (2003).

[71] A. Mishra and H. Mishra, Phys. Rev. D 69, 014014 (2004).

[72] S. B. Rüster and D. H. Rischke, Phys. Rev. D 69, 045011 (2004).

[73] M. Huang and I. Shovkovy, Nucl. Phys. A 729 (2003) 835.

[74] J.F. Liao and P.F. Zhuang, Phys. Rev. D 68, 114016 (2003).

[75] M. Alford, C. Kouvaris, and K. Rajagopal, Phys. Rev. Lett. 92, 222001 (2004).

[76] M. Alford, C. Kouvaris, and K. Rajagopal, hep-ph/0406137.

[77] K. Iida, T. Matsuura, M. Tachibana, and T. Hatsuda, Phys. Rev. Lett. 93, 132001 (2004).

[78] S. B. Rüster, I. A. Shovkovy, and D. H. Rischke, Nucl. Phys. A743, 127 (2004).

[79] K. Fukushima, C. Kouvaris and K. Rajagopal, hep-ph/0408322.

[80] S. Reddy and G. Rupak, nucl-th/0405054.

[81] W.V. Liu and F. Wilczek, Phys. Rev. Lett. 90, 047002 (2003).

[82] B. Deb, A. Mishra, H. Mishra, and P.K. Panigrahi, Phys. Rev. A 70, 011604(R) (2004).

[83] W. V. Liu, F. Wilczek, and P. Zoller, cond-mat/0404478

[84] M. M. Forbes, E. Gubankova, W. V. Liu, and F. Wilczek, hep-ph/0405059

[85] E. Gubankova, F. Wilczek, and E. G. Mishchenko, cond-mat/0409088

[86] F. Neumann, M. Buballa, and M. Oertel, Nucl. Phys. A714, 481 (2003). 
[87] P.F. Bedaque, H. Caldas, and G. Rupak, Phys. Rev. Lett. 91, 247002 (2003); H. Caldas, Phys. Rev. A 69, 063602 (2004).

[88] A. Gerhold and A. Rebhan, Phys. Rev. D 68, 011502 (2003); A. Kryjevski, Phys. Rev. D 68, 074008 (2003); D. D. Dietrich and D. H. Rischke, Prog. Part. Nucl. Phys. 53, 305 (2004).

[89] R. L. Stratonovich, Doklady Akad. Nauk S.S.S.R. 115, 1097 (1957); [translation: Sov. Phys. Doklady 2, 416 (1958)]; J. Hubbard, Phys. Rev. Lett. 3, 77 (1959).

[90] M. Huang and I. A. Shovkovy, Phys. Rev. D 70, 051501(R) (2004); hep-ph/0408268.

[91] D. H. Rischke, Phys. Rev. D 62, 034007 (2000); D. H. Rischke and I. A. Shovkovy, Phys. Rev. D 66, 054019 (2002).

[92] H. Muther and A. Sedrakian, Phys. Rev. D 67, 085024 (2003).

[93] A. I. Larkin and Yu. N. Ovchinnikov, Sov. Phys. JETP 20, 762 (1965); P. Fulde and R. A. Ferrell, Phys. Rev. 135, A550 (1964).

[94] M. G. Alford, J. A. Bowers, and K. Rajagopal, Phys. Rev. D 63, 074016 (2001); J. A. Bowers, J. Kundu, K. Rajagopal, and E. Shuster, Phys. Rev. D 64, 014024 (2001); R. Casalbuoni, R. Gatto, M. Mannarelli, and G. Nardulli, Phys. Rev. D 66, 014006 (2002); I. Giannakis, J. T. Liu, and H.-c. Ren, Phys. Rev. D 66, 031501 (2002); J. A. Bowers and K. Rajagopal, Phys. Rev. D 66, 065002 (2002).

[95] K. Rajagopal and F. Wilczek, Phys. Rev. Lett. 86, 3492 (2001).

[96] N. K. Glendenning, Phys. Rev. D 46, 1274 (1992).

[97] F. Weber, Pulsars as Astrophysical Laboratories for Nuclear and Particle Physics (Institute of Physics, Bristol, 1999).

[98] H. Heiselberg, C. J. Pethick, and E. F. Staubo, Phys. Rev. Lett. 70, 1355 (1993); N. K. Glendenning and S. Pei, Phys. Rev. C 52, 2250 (1995); N. K. Glendenning and J. Schaffner-Bielich, Phys. Rev. Lett. 81, 4564 (1998). 
[99] T. Norsen and S. Reddy, Phys. Rev. C 63, 065804 (2001); D. N. Voskresensky, M. Yasuhira, and T. Tatsumi, Nucl. Phys. A 723, 291 (2003).

[100] P. Slane, D.J. Helfand, and S.S. Murray, Astrophys. J. 571, L45 (2002).

[101] J.J. Drake, et al., Astrophys. J. 572, 996 (2002).

[102] D.G. Yakovlev, A.D. Kaminker, P. Haensel, and O.Y. Gnedin, Astron. 65 Astrophys. 389, L24 (2002).

[103] F.M. Walter and J.M. Lattimer, Astrophys. J. 576, L145 (2002).

[104] D. K. Hong and S. D. H. Hsu, Phys. Rev. D 68, 034011 (2003).

[105] S. Hands, I. Montvay, S. Morrison, M. Oevers, L. Scorzato, and J. Skullerud, Eur. Phys. J. C 17, 285 (2000); J. B. Kogut, D. K. Sinclair, S. J. Hands, and S. E. Morrison, Phys. Rev. D 64, 094505 (2001).

[106] K. G. Wilson, Phys. Rev. D 10, 2445 (1974); M. Creutz, Phys. Rev. D 21, 2308 (1980).

[107] F. Karsch, E. Laermann, and A. Peikert, Nucl. Phys. B 605, 579 (2001); M. Asakawa, T. Hatsuda, and Y. Nakahara, Prog. Part. Nucl. Phys. 46, 459 (2001); F. Csikor, Z. Fodor, S. D. Katz, and T. G. Kovacs, JHEP 0311, 070 (2003).

[108] F. Karsch, Nucl. Phys. Proc. Suppl. 83, 14 (2000); Z. Fodor and S. D. Katz, Phys. Lett. B 534, 87 (2002); JHEP 0203, 014 (2002); C. R. Allton et al., Phys. Rev. D 66, 074507 (2002).

[109] M. A. Halasz, A. D. Jackson, R. E. Shrock, M. A. Stephanov, and J. J. M. Verbaarschot, Phys. Rev. D 58, 096007 (1998); M. A. Stephanov, K. Rajagopal, and E. V. Shuryak, Phys. Rev. Lett. 81, 4816 (1998); P. de Forcrand and O. Philipsen, Nucl. Phys. B 642, 290 (2002).

[110] K. Rajagopal, Nucl. Phys. A 661, 150 (1999). 